\title{
Ciencia para la sustentabilidad: investigación, educación y procesos participativos
}

\author{
Sustainability science: research, education and participative processes
}

\author{
Alejandro Casas ${ }^{\text {a, } *}$, Ignacio Torres ${ }^{\text {a }}$, América Delgado-Lemus ${ }^{\text {a }}$, Selene Rangel-Landa ${ }^{a}$, \\ Catarina Ilsley ${ }^{\mathrm{b}}$, Juan Torres-Guevara ${ }^{\mathrm{c}}$, Aldo Cruz ${ }^{\mathrm{c}}$, Fabiola Parra ${ }^{\mathrm{c}}$, Ana Isabel Moreno-Calles ${ }^{\mathrm{d}}$, \\ Andrés Camou $^{\mathrm{d}}$, Alicia Castillo ${ }^{\mathrm{a}}$, Bárbara Ayala-Orozco ${ }^{\mathrm{a}}$, José J. Blancas ${ }^{\mathrm{e}}$, Mariana Vallejo ${ }^{\mathrm{f}}$, \\ Leonor Solís $^{\mathrm{a}}$, Atenea Bullen ${ }^{\mathrm{a}}$, Tamara Ortíz ${ }^{\mathrm{a}}$ y Berenice Farfán ${ }^{\mathrm{a}}$ \\ ${ }^{a}$ Instituto de Investigaciones en Ecosistemas y Sustentabilidad, Universidad Nacional Autónoma de México, Antigua Carretera a Pátzcuaro 8701, Col. \\ ExHacienda de San José de la Huerta, 58190 Morelia, Michoacán, México \\ ${ }^{\mathrm{b}}$ Grupo de Estudios Ambientales, A.C., Allende 7, Santa Úrsula Coapa, Del. Coyoacán, 04650 Ciudad de México, México \\ c Centro de Investigaciones en Zonas Áridas, Universidad Nacional Agraria La Molina, Camilo Carrillo 300-A, 11, Lima, Perú

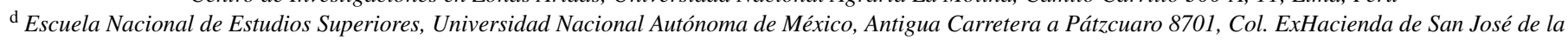 \\ Huerta, 58190 Morelia, Michoacán, México

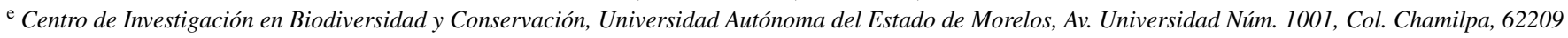 \\ Cuernavaca, Morelos, México

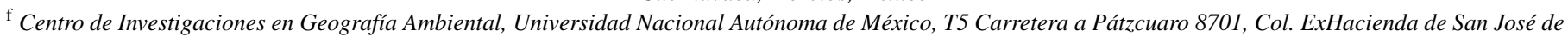 \\ la Huerta, 58190 Morelia, Michoacán, México \\ Recibido el 8 de marzo de 2016; aceptado el 8 de agosto de 2016 \\ Disponible en Internet el 23 de noviembre de 2017
}

\section{Resumen}

La ciencia para la sustentabilidad plantea incluir en investigaciones y acciones los conocimientos y técnicas desarrollados por diversos sectores de la sociedad. Esta propuesta reconoce que la complejidad de los problemas ambientales rebasa los enfoques y ritmos de la investigación científica predominante y brinda la posibilidad de acortar tiempos para la acción, aprovechando integralmente la experiencia humana. Presentamos un panorama de nuestro trabajo en: 1) investigación participativa, 2) procesos educativos para la participación y 3) procesos institucionales de comunicación y participación. Se abordan estudios socioecológicos participativos que involucran innovación tecnológica e intercambios de experiencias técnicas y organizativas para atender problemas ambientales en el valle de Tehuacán, la sierra Tarahumara, la montaña de Guerrero y la costa sur de Jalisco, poniendo énfasis en la construcción de alternativas. Se muestra la experiencia de prácticas de investigación de alumnos de posgrado y de la licenciatura en Ciencias Ambientales, UNAM. Ambos programas abordan problemas concretos en comunidades rurales en colaboración con organizaciones civiles y sociales, y comunidades. Se resumen las propuestas de vinculación institucional del Instituto de Investigaciones en Ecosistemas y Sustentabilidad (IIES) para atender problemas ambientales con manejadores de ecosistemas y tomadores de decisiones. Se discute la importancia de fortalecer procesos participativos como base para impulsar la colaboración académica en la construcción de perspectivas socioecológicas sustentables.

(C) 2017 Universidad Nacional Autónoma de México, Instituto de Biología. Este es un artículo Open Access bajo la licencia CC BY-NC-ND (http://creativecommons.org/licenses/by-nc-nd/4.0/).

Palabras clave: Ciencia para la sustentabilidad; Comunicación; Educación; Investigación participativa; Vinculación social

\footnotetext{
* Autor para correspondencia.

Correo electrónico: acasas@cieco.unam.mx (A. Casas).
}

La revisión por pares es responsabilidad de la Universidad Nacional Autónoma de México. 


\begin{abstract}
Sustainability science proposes including in research and actions knowledge and management techniques constructed by different sectors of the society. Such proposal recognizes that complexity of environmental problems exceeds approaches and rhythms of the predominant scientific research and provides the possibility of shortening time for action, making use of integral human experience. This work reviews a panorama of our work on: 1) participative-action research, 2) educational processes for participation, and 3) institutional processes of communication and participation. We address socio-ecological studies based on participative-actions involving technological innovation and exchange of technical and organizational experiences for solving environmental problems in Valle de Tehuacán, Sierra Tarahumara, Montaña de Guerrero, and the southern coast of Jalisco, emphasizing the construction of alternatives. We also show our experiences of participative research with students of graduate programs and the Environmental Sciences undergraduate program at UNAM. In both cases, we direct our studies on real environmental problems in rural contexts collaborating with NGOs and communities. We finally summarize our view on proposals of institutional linking of the Institute of Research on Ecosystems and Sustainability (IIES) for assessing environmental problems along with ecosystem managers and decision makers. We discuss the importance of strengthening participative processes as a platform for enhancing the academic collaboration for constructing sustainable socio-ecological perspectives.
\end{abstract}

(C) 2017 Universidad Nacional Autónoma de México, Instituto de Biología. This is an open access article under the CC BY-NC-ND license (http://creativecommons.org/licenses/by-nc-nd/4.0/).

Keywords: Sustainability science; Communication; Education; Participative research; Social vinculation

\section{Introducción}

La ciencia para la sustentabilidad es un campo de investigación que en las últimas décadas ha crecido exponencialmente en las publicaciones científicas del mundo (fig. 1). Surge de reconocer las limitaciones de los enfoques científicos y tecnológicos convencionales contemporáneos para entender y atender la grave crisis ambiental a escala planetaria (Kates et al., 2001). Tal crisis tiene múltiples expresiones socioecológicas, destacando lo que Peter Vitousek y numerosos académicos han denominado cambio global (Vitousek, 1994; Vitousek, D’Antonio, Loope y Westbrooks, 1996; Vitousek, Mooney, Lubchenco y Melillo, 1997). Cambios que incluyen el calentamiento promedio de la superficie de la Tierra, importantes alteraciones en ciclos del C, $\mathrm{N}$ y $\mathrm{P}$, la contaminación de los cuerpos de agua dulce y la de los mares por substancias tóxicas, metales pesados y materiales de lenta degradación, la pérdida acelerada de cobertura forestal y el abatimiento de la productividad primaria planetaria, las invasiones biológicas, y las elevadas tasas de extinción de especies y de variabilidad genética en poblaciones naturales y cultivos. En la dimensión humana, los cambios globales tienen sus expresiones más dramáticas en el crecimiento absoluto y relativo de la pobreza, el aumento de la desigualdad (OXFAM, 2012), y la concentración acelerada de poder político y económico. Los procesos migratorios del campo a la ciudad y del sur al norte globales, los conflictos religiosos e interétnicos, genocidios, el gigantesco negocio de la minería, el petróleo y derivados, las drogas, armas y lavado de dinero a múltiples escalas (Buscaglia, 2015), la pérdida de lenguas y culturas (Lewis, Gary, Simons y Fennig, 2015), así como la pérdida de patrimonio biocultural (Maffi, 2007, Maffi y Woodley, 2010; Boege, 2008; Toledo y Barrera-Bassols, 2008), son todas expresiones de la crisis global en contextos socioculturales.

El Millennium Ecosystem Assessment (MEA, 2005) aportó un importante diagnóstico del grado de deterioro de los ecosistemas del planeta. Steffen, Broadgate, Deutsch, Gaffney y Ludwig (2015) y Steffen, Crutzen y McNeill (2007) coinciden en que la drástica transformación a escala planetaria debida a actividades humanas ha llevado a la Tierra a una nueva era geológica, el Antropoceno. El gran drama de estos procesos es la brevedad del lapso en que han ocurrido los cambios. Estudios recientes indican que la tasa de daño severo a los ecosistemas ha crecido de manera acelerada en los últimos 65 años (Barnosky et al., 2012); es decir, a partir del impulso y consolidación de los modelos de desarrollo global que surgieron después de la Segunda Guerra Mundial. Estos incluyen la globalización de mercados, la obsesión por el crecimiento económico, la tecnología dirigida a intensificar la productividad, la ciencia integrada a la producción y el consumismo de bienes inútiles como base de la activación de los mercados (Scott, 1996; Martínez-Alier, 2015). Tal apuesta de desarrollo ha tenido consecuencias ambientales catastróficas en unas cuantas décadas (Scott, 1996). Existen procesos como el de la minería a cielo abierto que en cuestión de pocos años, menos de una década, tienen una alta capacidad destructiva de sistemas socioecológicos y en elementos bioculturales moldeados por siglos o milenios.

Los acuerdos y acciones locales y globales para atender la magnitud de los problemas ambientales multicausales se desenvuelven a ritmos excesivamente lentos para la rapidez con la que ocurre la destrucción. La ciencia para la sustentabilidad adquiere entonces un carácter paradigmático que busca la posibilidad de proyectar a futuro los procesos que sostienen la vida en el planeta, la permanencia de las sociedades y de nuestra propia especie. La ciencia y la sociedad actuales están en una carrera contra el tiempo y las acciones requieren pasos firmes y ágiles para lograr metas a corto plazo. Lo que ha ocurrido en 65 años requiere ya acciones que tomarán siglos para reparar procesos alterados. El viraje drástico en el modelo de desarrollo hegemónico es una necesidad impostergable, pues pone en entredicho la perspectiva futura de la vida en el planeta.

Las premisas de la ciencia para la sustentabilidad se han discutido ampliamente desde los primeros intentos por delinear su perfil. Sus bases conceptuales comenzaron a desarrollarse en la segunda mitad de la década de 1990, y una de las publicaciones más influyentes es la de Kates et al. (2001), y posteriormente las de Clark y Dickson (2003), Clark (2007) y Kates (2011), 


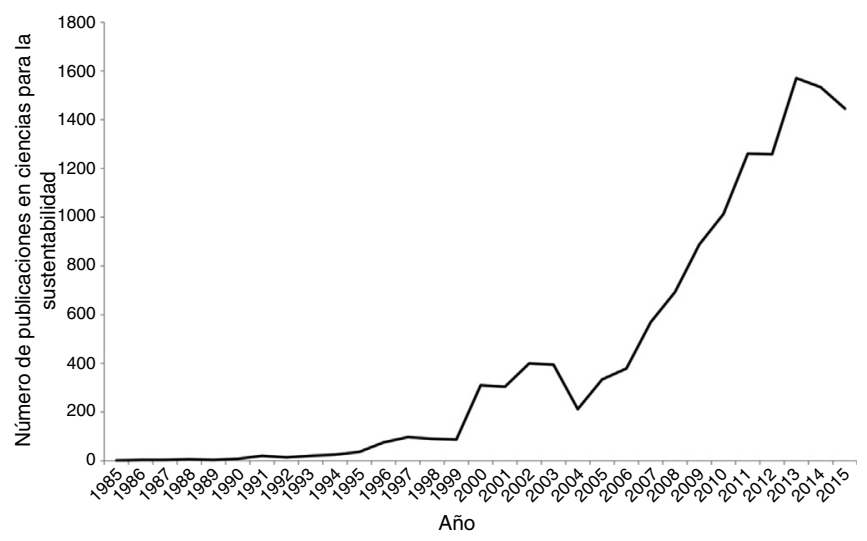

Figura 1. Incremento en el número de publicaciones científicas que incluyen entre sus palabras clave «ciencia para la sustentabilidad» con base en la información almacenada en la base de datos de SCOPUS.

entre otros cada vez más numerosos trabajos. Estos autores coinciden en que la ciencia para la sustentabilidad constituye un paradigma, un campo emergente de investigación para entender las interacciones entre naturaleza y sociedad, estrechamente ligado a la acción para afrontar la crisis ambiental global. Este campo requiere la integración de diversas áreas científicas bajo enfoques interdisciplinarios sensu García (1994), debido a la multiplicidad de factores causales involucrados en el proceso, así como la valoración e inclusión del conocimiento de diversos sectores de la sociedad, como por ejemplo el conocimiento ecológico tradicional sensu Alcorn (1993), Berkes (1999), Berkes y Berkes (2009), Berkes, Colding y Folke (2000), Toledo (2002), cuya visión frecuentemente es más amplia que la incipiente investigación científica de algunos procesos. Esta visión científica plantea la necesidad de trabajar con distintos sectores sociales y transitar a lo que se ha denominado transdisciplina, sensu Gibbons et al. (1994); Klein et al. (2001), Lang et al. (2012); Scholz y Steiner (2015), entre otros autores. Se requieren nuevas concepciones de construcción y uso del conocimiento, más amplias y flexibles que las que predominan en las ciencias convencionales (Funtowicz y Ravetz, 1993), nuevas estrategias de investigación e innovación, así como adecuaciones institucionales para lograrlo (Cornell et al., 2013).

Entre los principales rasgos de la ciencia para la sustentabilidad que se han propuesto destacan: 1) la necesidad de visualizar y comprender los procesos de interacción entre naturaleza y sociedad, bajo una visión integrada de procesos socioecológicos mutuamente influyentes; 2) el reconocimiento de tales procesos como fenómenos sistémicos complejos y la importancia de entender los componentes y propiedades de tales sistemas (e. g. propiedades emergentes, incertidumbre, adaptabilidad, estabilidad, resiliencia, autoorganización), para comprender la sustentabilidad como un proceso dinámico, de construcción continua; 3) la comprensión de los sistemas socioecológicos a escalas locales, regionales y globales y las influencias recíprocas entre estos (transescalaridad); 4) la necesidad de desarrollar estrategias de investigación multidisciplinar, interdisciplinar y transdisciplinar, capaces de detonar interacciones entre distintas áreas de la investigación científica para entender las diferentes aristas de un problema en común, y 5) establecer bases institucionales sólidas para compartir los saberes y experiencias interdisciplinarias y multisectoriales sobre los problemas ambientales, con el fin de aprovecharlos y actuar de manera concertada en la atención de problemas.

La ciencia para la sustentabilidad reconoce la importancia de la investigación interdisciplinaria de distintas áreas de la ciencia para atender preguntas y problemas bajo marcos epistémicos en común (García, 1994). Identifica la importancia de la transdisciplina como parte del enfoque de investigación y de acción, capaz de involucrar conocimiento y procesos de construcción de conocimientos e innovación tecnológica de grupos e individuos de distintos sectores de la sociedad (e.g., grupos indígenas, asociaciones de productores, organizaciones civiles, entidades gubernamentales, empresas) en un diálogo concertado con los grupos académicos. Para hacer frente a los cambios ambientales mencionados, tales sectores desarrollan continuamente estrategias y conocimientos para adaptarse a las nuevas condiciones, de modo que fortalecer e integrar los sistemas de conocimiento de los distintos sectores es fundamental para la construcción de sistemas socioecológicos firmes (Tengö, Brondizio, Elmqvist, Malmer y Spierenburg, 2014). Kates et al. (2001) destacan que la ciencia para la sustentabilidad requiere estrategias de investigación que reconozcan y valoren el amplio rango de visiones que permitan hacer utilizable el conocimiento generado tanto por la ciencia como por la sociedad. Destacan la importancia de visualizar que en una sociedad de riesgo global es de excepcional importancia la interacción entre científicos, tomadores de decisiones de diferentes ámbitos (gubernamental, productivo, empresarial) y, en general, con los ciudadanos, quienes pueden utilizar el conocimiento para entender los problemas de manera más completa y modificar aquellas acciones que les correspondan. Dada la complejidad de los sistemas socioecológicos, la colaboración entre diversos actores y la integración de los conocimientos locales se vuelven clave en la construcción de un entendimiento de los sistemas y de sus posibles respuestas a los cambios. Más importante aún, la participación de diversos actores permite avanzar hacia un proceso inclusivo, en el que la investigación no es solo un medio para descubrir sino también parte de un proceso social y político (Clark, Lorrae van Kerkhoff y Gallopin, 2016).

El diálogo de saberes, el intercambio de experiencias técnicas y prácticas para la comprensión y atención de problemas es entonces un aspecto crítico de la ciencia para la sustentabilidad. $\mathrm{Y}$ en buena medida, su importancia radica en la posibilidad de acortar los tiempos para encontrar soluciones. En este trabajo analizamos experiencias de investigación en 3 esferas de trabajo que involucran vínculos entre la investigación académica y distintos sectores de la sociedad: 1) procesos de investigación participativa, 2) procesos educativos en el contexto de la ciencia para la sustentabilidad y 3) procesos institucionales para la vinculación de la ciencia con la sociedad. Presentamos en cada caso un panorama de experiencias y resultados y discutimos los retos principales para adecuar el quehacer científico en directrices que aporten a la construcción de ciencia para la sustentabilidad. 


\section{Procesos de investigación participativa}

El esquema predominante de investigación para atender problemas ha tenido un carácter lineal: la investigación genera conocimientos que se transforman en innovaciones tecnológicas y estas se transfieren a agentes sociales de distintos ámbitos: producción agrícola, ganadera, forestal, procesos industriales o áreas como salud y educación (Adames-Mayorga, 2007; Chambers, 1983; Röling, 1990; Van Kerkhoff y Lebel, 2006). Ha sido un modelo ampliamente utilizado en la llamada revolución científico-técnica, la cual ha tenido grandes éxitos pero también extraordinarios fracasos. Este esquema de innovación tecnológica ha contribuido a acelerar drásticamente los problemas ambientales globales. Una crítica fundamental al modelo comenzó en la década de 1970 cuando el esquema de transferencia tecnológica implementado para el desarrollo agrícola de países en desarrollo, reconocido como Revolución Verde, fue fuertemente cuestionado por diversos autores. Entre ellos destacó el educador brasileño Paulo Freire (Freire, 1973), para quien el concepto de extensionismo, entendido como la acción de transmitir las innovaciones diseñadas por grupos de científicos o empresas es un proceso de imposición cultural que no solamente no resolvía los problemas enfrentados por los agricultores, sino que obstaculizaba las posibilidades de transformación social de grandes proporciones de trabajadores del campo. Freire cuestionó el proceso unidireccional debido a los múltiples fracasos de procesos tecnológicos, así como a graves problemas sociales y ecológicos que desató, además de detonar procesos de desvalorización de conocimientos, tecnologías y recursos locales. Resaltó la relevancia de impulsar procesos de comunicación dialógica bidireccional o multidireccional, contrapuestos al de extensión unidireccional (Freire, 1973; Altieri y Toledo, 2011). Freire puso énfasis no solo en la unidireccionalidad del proceso comunicativo sino, sobre todo, en el carácter político que este modelo de intervención campesina tuvo para ocultar las intenciones finales del paquete tecnológico de la Revolución Verde. Su crítica no era solo metodológica, denunció la pobreza educativa del extensionismo, al no buscar elevar el nivel de comprensión crítica sobre la realidad entre el campesinado y su empoderamiento. De manera que el pensamiento de Freire no solo comprende la crítica a su metodología, sino ante todo, a su carácter castrante en el mundo campesino.

\section{Procesos educativos para la sustentabilidad}

Desde los trabajos de Freire hasta nuestros días, se ha promovido una visión de la educación como un proceso a través del cual se comparten y construyen conocimientos por los involucrados (educadores y educandos) en proyectos de transformación socioecológica (Castillo, 1999). Se habla entonces de modelos de coproducción de conocimientos (Gibbons, 2000) que requieren organizaciones sociales flexibles para su mejor desarrollo. Un aspecto que emerge del trabajo comunicativo educativo es la toma de conciencia sobre la realidad que se vive en los sectores involucrados, y sobre todo, se reconocen las propias capacidades de las comunidades y de los otros sectores que trabajan en conjunto. Las personas que cooperan y colaboran se empoderan, e incrementan y fortalecen la participación social de los distintos sectores en la toma de decisiones.

\section{Procesos institucionales para la sustentabilidad}

La iniciativa y acción de los individuos en los procesos transformadores constituyen piedras angulares sobre las que descansan las metas para la solución de problemas socioecológicos. Pero sin duda las iniciativas individuales y aun grupales suelen ser limitadas. Una premisa fundamental de este trabajo es que las perspectivas deben involucrar cada vez más a las instituciones, los centros, institutos, universidades, ministerios, gobiernos, redes sociales. En la medida en que las iniciativas alcancen tal nivel de socialización podrán lograr metas de mayor trascendencia. En este sentido, las iniciativas individuales y grupales deben aspirar a convertirse en iniciativas institucionales y políticas que transiten de las reglas locales a políticas públicas.

En las siguientes secciones compartimos experiencias de investigación grupal e institucional y acciones educativas y participativas que han buscado a través del diálogo de saberes la construcción de conocimientos para la atención de problemas socioecológicos. Sobre la base de estas experiencias aspiramos a extraer algunas conclusiones que puedan ser de utilidad en el objetivo de reorientar la investigación científica para contribuir a la construcción de ciencia para la sustentabilidad.

\section{Agaves, bosques y mezcal}

El primer caso que ayuda a ilustrar la colaboración dialógica intersectorial se lleva a cabo en la comunidad de San Luis Atolotitlán, en el valle de Tehuacán (fig. 2). Con base en la confianza generada por cerca de 10 años de trabajo local, las autoridades ejidales solicitaron nuestra colaboración en un proyecto que iniciaron para recuperar poblaciones Agave potatorum (fig. 3), un maguey usado en la zona desde hace más de un siglo para elaborar mezcal de excelente calidad. Por mucho tiempo, la producción fue a pequeña escala, para satisfacer la demanda comunitaria en fiestas tradicionales; pero en las últimas 2 décadas aumentó su demanda en el mercado regional y con ello el número de fabricantes y de agaves extraídos de los bosques (Delgado-Lemus, Casas y Téllez, 2014). Después de reconocer su extinción en algunas localidades, la comunidad decidió recuperar los agaves en sus hábitats naturales con el acompañamiento técnico y gestión de apoyos por parte de las autoridades de la Reserva de la Biosfera Tehuacán-Cuicatlán (RBTC), de la Comisión Nacional de Áreas Naturales Protegidas (Conanp) (fig. 3).

Las autoridades ejidales recuperaron un vivero, organizaron la colecta de semillas, la producción de plantas de agave y su trasplante a áreas forestales. Después de un año apreciaron una alta mortandad de los individuos de agave trasplantados y fue entonces que pidieron nuestra asesoría. Junto con los productores se realizó una evaluación de la mortalidad de las plantas, identificando que esta era del $56 \%$ en tan solo un año. Iniciamos entonces un proceso de investigación acerca de las causas. Entre 


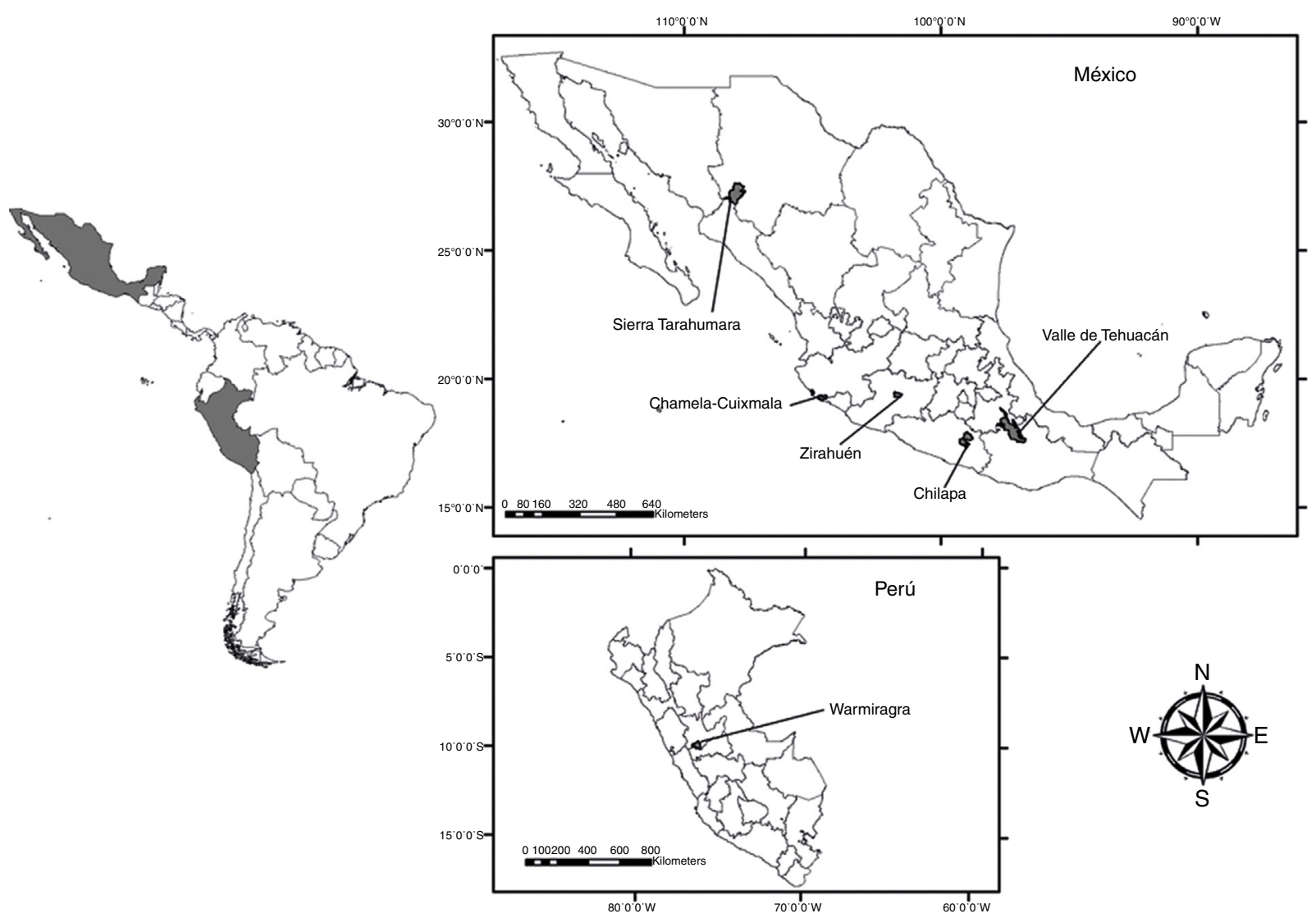

Figura 2. Sitios de estudio referidos en el artículo. Arriba, las regiones de México mencionadas en el texto; abajo la región de la Sierra Central Andina del Perú, en donde se encuentra la cuenca de Warmiragra, municipalidad de Tomaykichwa, en el Distrito de Huánuco, Perú.

las más importantes se identificó un problema asociado a interacciones de facilitación. Se investigó la distribución y abundancia del maguey en distintos tipos de vegetación y de identificaron en concreto sus interacciones de facilitación o de asociación a plantas nodrizas. La investigación al respecto (Rangel-Landa, Casas y Dávila, 2015) identificó una asociación significativa de A. potatorum con diferentes especies de plantas arbustivas, arbóreas y aun herbáceas en distintos tipos de vegetación y que tal asociación era determinante para el establecimiento de plántulas o agaves juveniles. Ello permitió recomendar el trasplante de agaves bajo el dosel de ciertas especies de plantas en particular. También se propuso realizar muestreos rápidos de nodrizas en distintos tipos de vegetación, pues A. potatorum se distribuye en al menos 14 tipos de asociaciones vegetales (Valiente-Banuet et al., 2009). El trabajo de Rangel-Landa et al. (2015) confirmó que el desempeño de las semillas y plántulas de un sitio determinado decrece en sitios distintos al del origen, lo que condujo a recomendar el control del origen de las semillas y trasplantar las plántulas al tipo de vegetación correspondiente. Las diferencias en los linajes de poblaciones de sitios distintos se confirmaron en el estudio de Félix-Valdés et al. (2015), quienes encontraron diferenciación genética entre poblaciones separadas por tan solo $3 \mathrm{~km}$, pero en condiciones ambientales distintas.

Mediante muestreos en el territorio de la comunidad, se estimó (Delgado-Lemus et al., 2014a; Delgado-Lemus, Torres, Blancas y Casas, 2014) que anualmente se extraen cerca del $65 \%$ de los individuos reproductivos de A. potatorum, aunque hay sitios en donde se extraen todos estos. Este hecho indicó la importancia de planear la extracción, asegurando la permanencia de individuos reproductivos en cada sitio de extracción. EstrellaRuiz (2008) encontró que la visita de murciélagos polinizadores a las flores de A. potatorum disminuía drásticamente en sitios en donde escaseaban los individuos reproductivos. Siendo que la entrecruza es obligada para la producción de semillas, la baja visita de polinizadores a los pocos individuos reproductivos determina que estos tengan una escasa producción de semillas. Un estudio demográfico (Torres, Casas, Delgado-Lemus y Rangel-Landa, 2013; Torres, Casas, Vega, Martínez-Ramos y Delgado-Lemus, 2015) permitió apreciar que aun las poblaciones más conservadas podrían extinguirse en un periodo de 23 años. Se identificaron las etapas críticas del estado de desarrollo de los agaves en los que es más eficiente llevar a cabo el repoblamiento de áreas forestales, o que complementarían las propuestas técnicas para un manejo más sustentable de la especie (fig. 3).

El proyecto promovió que las autoridades y encargados del vivero conocieran la experiencia de manejo forestal de maguey de la organización Sansekan Tinemi en Chilapa, Guerrero. Junto con la asociación civil el Grupo de Estudios Ambientales y Sociales (GEA) se organizaron intercambios de experiencias de manejo forestal en 2006 y en 2015. Los campesinos de Guerrero manejan Agave cupreata. Para ambas organizaciones fue muy enriquecedor conocer las técnicas desarrolladas en cada sitio y se detonó un intercambio de prácticas de innovación. En 


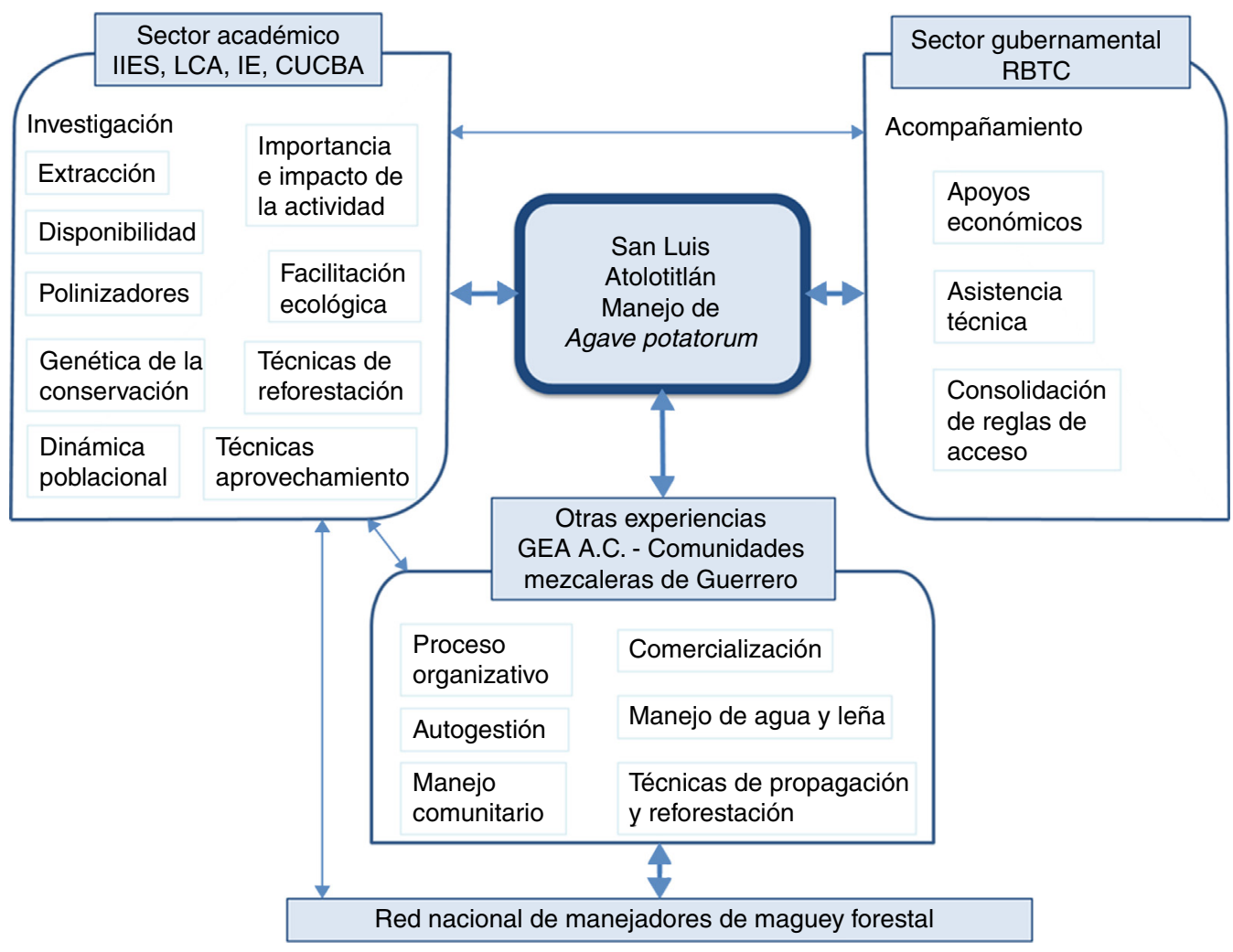

Figura 3. Relaciones intersectoriales en el proceso de construcción de nuevas técnicas de manejo de Agave potatorum. El diálogo de saberes (flechas) ha sido el eje de la interacción de la comunidad de San Luis Atolotitlán, los sectores académico y gubernamental y otras experiencias en el manejo forestal y cultivo de agaves mezcaleros del país. CUCBA: Centro Universitario de Ciencias Biológicas y Agropecuarias, Universidad de Guadalajara; GEA, A.C.: Grupo de Estudios Ambientales, Asociación Civil, IE: Instituto de Ecología, UNAM; IIES: Instituto de investigaciones en Ecosistemas y Sustentabilidad, UNAM; LCA: licenciatura en Ciencias Ambientales, UNAM; RBTC: Reserva de la Biosfera Tehuacán-Cuicatlán.

la comunidad de Tehuacán se difundió la información generada mediante: 1) reuniones con autoridades y responsables del vivero, 2) reuniones generales con la comunidad, y 3) talleres sobre producción, consumo y comercialización. Las autoridades de la RBTC y las asambleas comunitarias tomaron en cuenta los diagnósticos y recomendaciones para construir regulaciones para el acceso a los agaves (por ejemplo, sitios de veda, restricciones de acceso del ganado, áreas de reforestación). El proceso no ha terminado, otras comunidades de Tehuacán productoras de mezcal y otras organizaciones del país, incluyendo organizaciones de Jalisco y Michoacán, se han incorporado al proceso de intercambio de experiencias. Junto a la producción sustentable, los productores enfrentan problemas en la protección de derechos de propiedad intelectual y en la gestión de mercados justos. Estos temas requieren la participación de especialistas de otras áreas. La sustentabilidad del aprovechamiento de A. potatorum implica asegurar el aprovechamiento del recurso a largo plazo, pero también el adecuado beneficio económico y social de esta actividad (fig. 3).

\section{Manejadores rarámuri del bosque}

Un segundo trabajo se llevó a cabo en la sierra Tarahumara, en el estado de Chihuahua, México. Esta experiencia se desarrolló con la relación entre una ONG, la Consultoría Técnica Comunitaria (Contec), una comunidad rarámuri del ejido forestal de
Cuiteco y una institución académica, el Centro de Investigaciones en Ecosistemas (CIEco, ahora IIES), de la UNAM. Contec es una organización civil que trabaja en la sierra Tarahumara desde hace 15 años y tiene como misión apoyar procesos organizativos que favorezcan la economía campesina y la gobernabilidad local de las comunidades agrarias en la sierra Tarahumara. Desarrolla su trabajo con base en una estrategia de educación no formal que busca responder a las necesidades y temáticas de interés de las comunidades participantes.

El ejido de Cuiteco desarrolló la extracción de madera para la industria forestal con fines comerciales entre 1981 y 2002, año a partir del cual, por acuerdo comunitario, la extracción de madera dejó de practicarse, entre otras razones debido a que los bajos ingresos que obtenían no compensaban el alto costo ambiental y laboral de los campesinos involucrados. El manejo forestal suscitó procesos de extracción irregular de madera, lo que derivó en la interposición de denuncias ante la Procuraduría Federal de Protección al Ambiente (Profepa) por parte de los mismos campesinos (CCA, 2005). Ante tal panorama, la comunidad rarámuri optó por la búsqueda de opciones productivas asociadas al manejo sustentable de los recursos forestales no maderables (RFNM) y la conservación del bosque (Comisariado Ejidal de Cuiteco, 2007).

Ante la solicitud de colaboración de la comunidad hacia nuestro grupo, nuestra participación estuvo dirigida principalmente a inventariar los recursos potenciales y a analizar cómo 
A

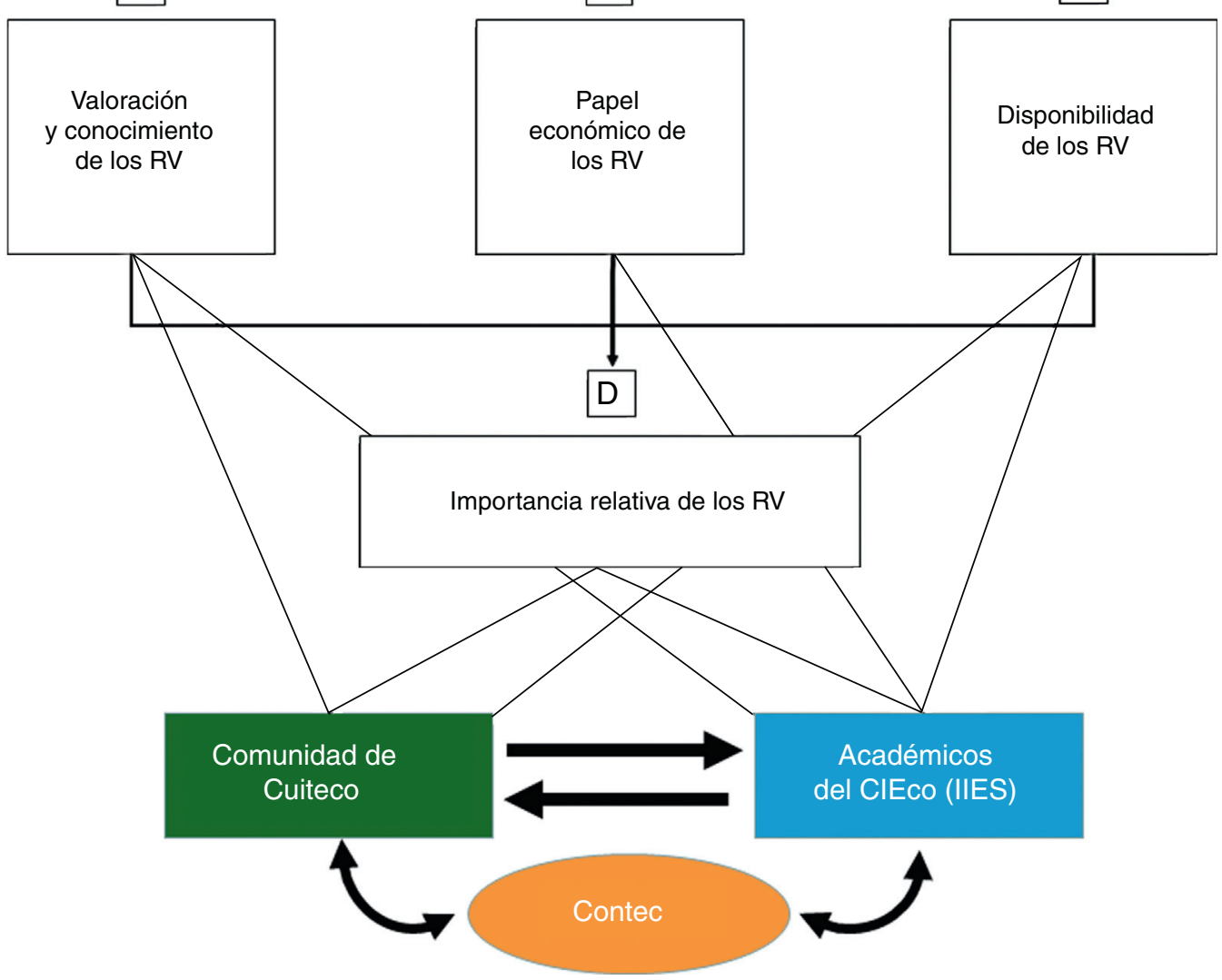

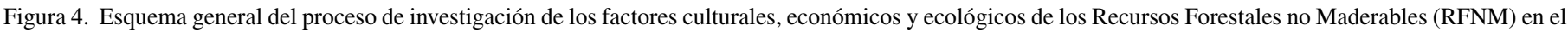

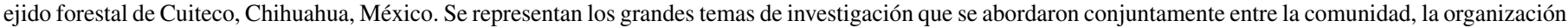

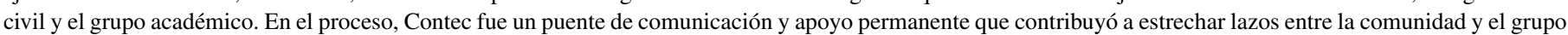

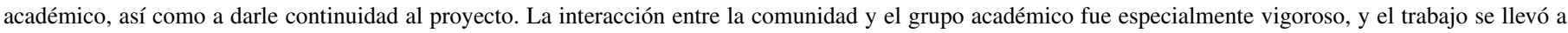
cabo con equipos mixtos de trabajo entre campesinos y académicos.

influyen factores culturales, económicos y ecológicos, en las formas de aprovechamiento de los RFNM en la comunidad forestal, con el fin de entender las decisiones y técnicas adoptadas para aprovechar los RFNM y generar propuestas para su manejo sustentable. Para cumplir tales objetivos se implementó una estrategia de Investigación Acción Participativa, a través de la cual: 1) se identificaron las unidades que componen el territorio del ejido, 2) se diseñó una estrategia para la elaboración de un inventario etnobotánico, 3) se elaboró un estudio ecológico que aportó información sobre la distribución y abundancia de los recursos vegetales como criterio para identificar la viabilidad de su aprovechamiento comercial y 4) se elaboró un estudio sobre la importancia económica de los recursos vegetales (Camou-Guerrero, Reyes-García, Martínez-Ramos y Casas, 2008) que permitió identificar aquellos recursos con mayor potencial (fig. 4). Un esquema de investigación similar al de nuestro grupo lo había ensayado en otras comunidades del país. La particularidad de este proyecto es que se construyó en conjunto con la ONG y la comunidad, y esta última se apropió del proceso de manera destacada. El trabajo se desarrolló conjuntamente con un equipo técnico campesino y el acompañamiento de la asamblea ejidal, complementando y validando la información generada (fig. 5). Algunas de las aplicaciones directas de este proceso de investigación han sido: 1) el inventario de recursos forestales no maderables generado, el cual permitió impulsar la formación de una cooperativa para la elaboración de productos a base de plantas locales; 2) se generó una tipología de recursos la cual permitió orientar estrategias de manejo de RFNM, como la protección de recursos vulnerables debido a su escasez e intensidad de uso; o el aprovechamiento de recursos potenciales por su valor comercial y adecuada abundancia; y 3) la delimitación y establecimiento de una Reserva Campesina para la conservación de la biodiversidad (CamouGuerrero, 2008). En esta experiencia es de particular relevancia el esfuerzo empleado para generar mecanismos de comunicación entre los 3 actores involucrados, para la definición de los objetivos y métodos de la investigación, así como diseñar las acciones derivadas.

\section{Investigación desde la perspectiva de los actores sociales en la costa sur de Jalisco}

La UNAM creó en 1971 la Estación de Biología Chamela (Noguera, Vega y Aldrete, 2002) con el objetivo de proteger el área e investigar los componentes y funcionamiento de los ecosistemas de bosque tropical seco. Durante más de 4 décadas, la producción científica rebasa ya los 700 artículos científicos y 500 tesis, y es el sitio con más estudios del bosque tropical 

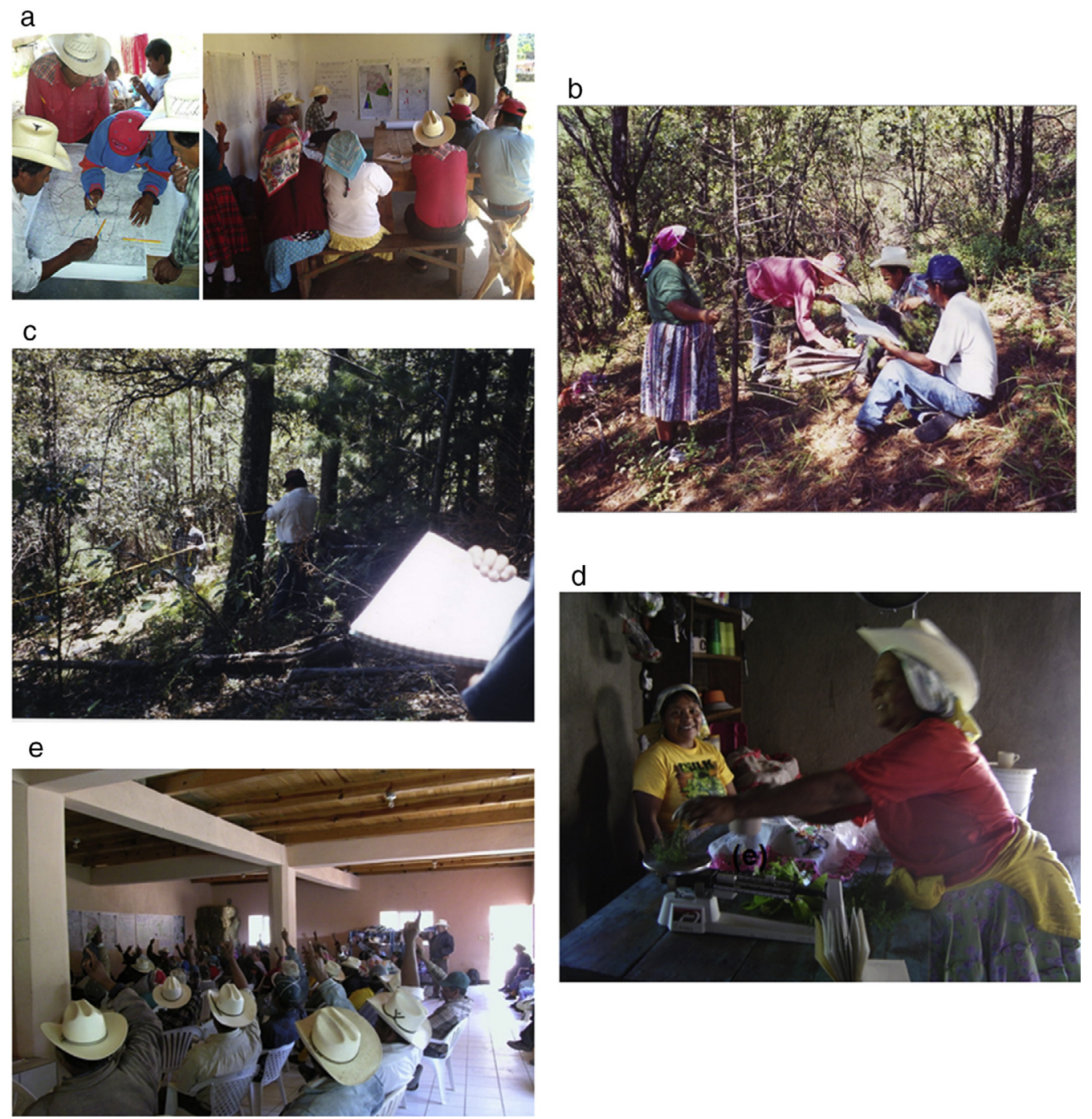

Figura 5. 5(a), La zonificación y caracterización del territorio se realizó mediante un proceso de mapeo participativo, para lo cual se desarrollaron talleres en los cuales participó un equipo técnico campesino conformado por hombres y mujeres. El proceso de mapeo participativo incluyó la presentación de la cartografía resultante en la asamblea ejidal para su socialización y validación. La cartografía temática generada incluyó: tipos de vegetación, usos del suelo y unidades ambientales o de manejo; 5(b), el equipo técnico campesino desarrolló un inventario etnobotánico partiendo de los diferentes tipos de vegetación y unidades ambientales. El inventario etnobotánico, conjuntamente con la cartografía comunitaria fue la base de análisis más detallados que permitieron derivar recomendaciones y estrategias de manejo de los RVNM. La información de nomenclatura y formas de uso de los ejemplares colectados fueron recopilados y sistematizados igualmente en talleres etnobotánicos y en asamblea ejidal; 5(c), el equipo técnico campesino se capacitó para la realización de muestreos de vegetación, siguiendo básicamente el método de muestreo propuesto por Gentry. Con el propósito de determinar los patrones de distribución y abundancia de los RVNM en el ejido Cuiteco. Se formaron 4 equipos de campesinos y ecólogos que lograron efectuar cerca de 30 puntos de muestreo en una semana (lo que nuestro grupo trabajando solo logra en más de 3 meses de trabajo); 5(d), el análisis económico de los RVNM (valor de uso y valor monetario) se desarrolló en una muestra representativa de las familias rarámuri de Cuiteco. Incluyó la aplicación de encuestas y mediciones directas de consumo de RVNM; 5(e), asamblea ejidal en el ejido Cuiteco para la validación del trabajo de ordenamiento de las actividades de manejo de los RVNM.

seco del continente (Noguera et al., 2002). Sin embargo, el uso de esta información para promover el aprovechamiento sustentable de recursos naturales ha sido mínimo (Castillo, Pujadas, Magaña, Martínez y Godínez, 2006; Pérez-Escobedo, 2011), no obstante que es altamente necesario para los habitantes de las comunidades aledañas. Considerando tal situación, desde el año 2000 se iniciaron estudios para documentar las visiones de los pobladores locales sobre el uso y manejo de sus tierras y los temas prioritarios de manejo ambiental.

Se partió de un enfoque de investigación similar al que Gibbons (2000) llama «Modo 2» de generación de conocimientos. Este modelo es de carácter aplicado y se diferencia del Modo 1 en el cual las agendas de investigación las deciden los científicos. El Modo 2 busca que las agendas y preguntas de investigación se basen en problemas identificados por actores sociales no científicos; esto es, personas o grupos sociales que requieran información científica para entender y resolver o mitigar problemas específicos que les atañen (fig. 6).

Con base en este enfoque y a través de perspectivas cualitativo-interpretativas de investigación (Tarrés, 2004) con técnicas como la observación participante, la conducción de entrevistas a profundidad y talleres participativos, se han 


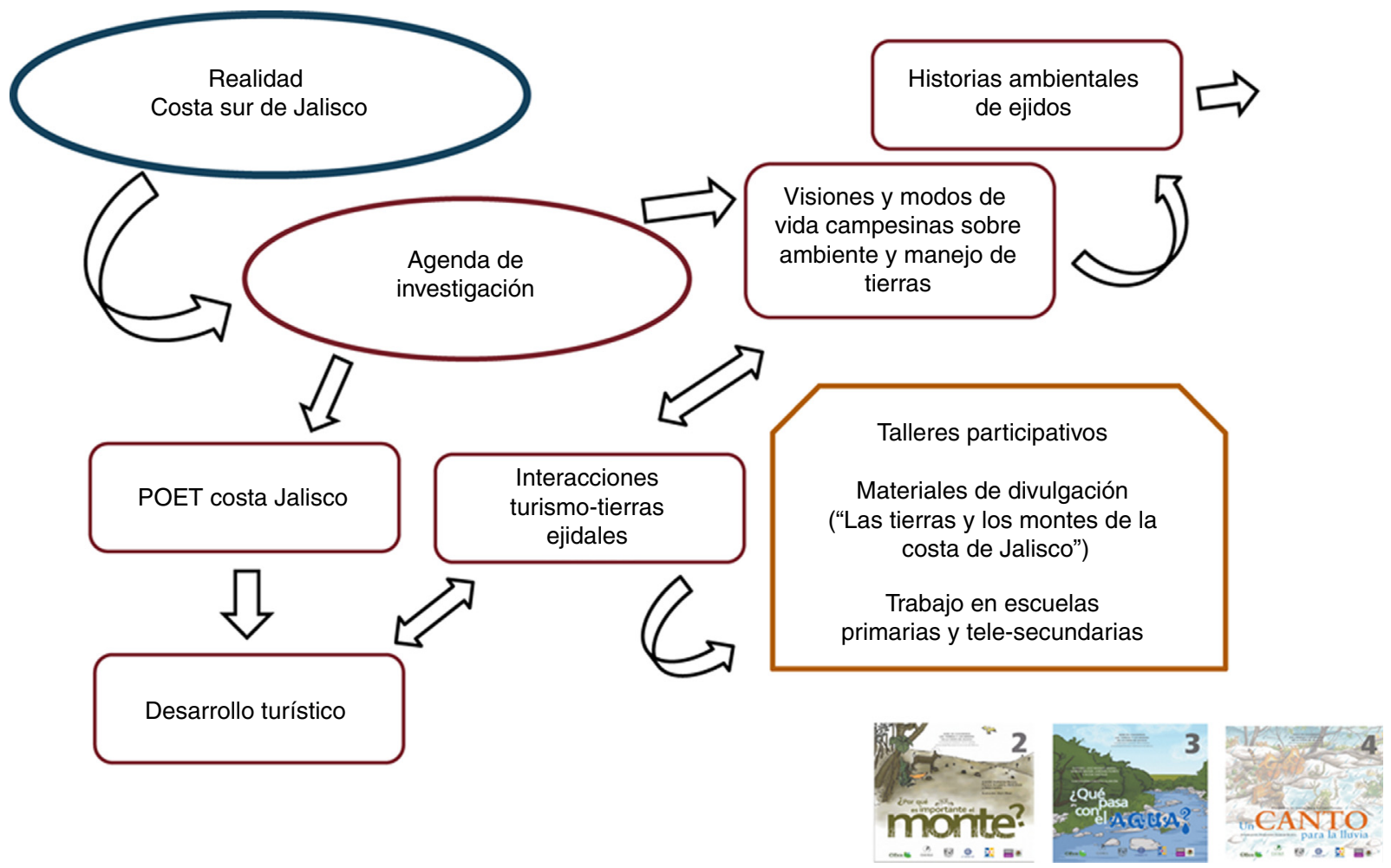

Figura 6. Enfoque de trabajo seguido en la costa sur de Jalisco. Se pone énfasis en 2 grandes temas de extrema relevancia: el manejo de tierras y los modos de vida campesinos, así como el desarrollo turístico en la actualidad (impulsado por el Programa de Ordenamiento Ecológico Territorial [POET]). Estos temas se han trabajado en talleres participativos con familias campesinas, así como a través del uso de materiales educativos.

identificado temas relevantes de interés local y regional. Un ejemplo está asociado al proceso de formulación del «Ordenamiento Ecológico Territorial de la Costa de Jalisco» decretado en 1999, el cual fue fuertemente rechazado por las poblaciones locales. Los análisis realizados permitieron documentar las fallas en la formulación del programa, principalmente en relación con la consulta pública. En voz de los participantes, «en teoría era para ver si la gente estaba o no de acuerdo en intercambiar opiniones, pero acabó siendo una reunión informativa, no se tuvo la intención de tomar en cuenta a nadie» (Pujadas, 2003). A la fecha, este instrumento de política ambiental se ha utilizado para justificar el desarrollo de proyectos turísticos. Por ello, el turismo se identificó como un tema prioritario.

Hasta ahora no se ha identificado un fuerte impacto ambiental debido al turismo; sin embargo, en los últimos años se han aprobado varios proyectos turísticos de grandes dimensiones que sí provocarán impactos negativos en la región. Desde 2007, académicos de la UNAM hemos realizado reportes científicos, que demuestran que las propuestas deben modificarse para evitar daños ecológicos (Boege et al., 2010; Castillo, Domínguez, García, Quesada y Vega, 2007). Sin embargo, y a pesar de que la Conanp ha avalado las sugerencias de la UNAM, otras instancias gubernamentales han otorgado permisos y los proyectos están en marcha. El estudio del turismo en la región revela la existencia de una fuerte asimetría ambiental. Por ejemplo, en cuanto al acceso al agua, un recurso común escaso, una de las mansiones de la zona paga 1,700 dólares por 1 millón de litros de agua al año mientras que un campesino paga 7,400 dólares por la misma cantidad de agua que alcanza para mantener
40 vacas durante 21 meses (Cohen, 2014; Riensche, Castillo, Flores-Díaz y Maass, 2015). Otra muestra de la asimetría es que el agua que se consume en la zona adyacente al mar se obtiene de pozos, cuyos mantos freáticos se recargan por la presencia de vegetación en las tierras campesinas ubicadas tierra adentro (fig. 7). Los dueños de desarrollos turísticos obtienen beneficios económicos mientras que las comunidades campesinas locales no (Riensche et al., 2015).

Otro ejemplo de procesos para identificar los temas de investigación relevantes para los actores sociales, lo brinda la historia ambiental (fig. 6). En la región, la unidad de análisis han sido los ejidos, pues poseen el $70 \%$ de las tierras del municipio La Huerta en donde se localiza la Estación Chamela de la UNAM (Castillo et al., 2009). Se documentaron las visiones y modos de vida de las familias campesinas. Debido a que los ejidos en esta parte de México fueron de los últimos decretados por la Reforma Agraria, fue posible conocer a los fundadores de ejidos y entrevistarlos. Además de conocer los sucesos que permiten entender las decisiones actuales sobre el manejo de ecosistemas, la reconstrucción de las historias ambientales de los ejidos se escribió y se elaboraron artesanalmente pequeños libros que fueron entregados a los ejidos. Los libros fueron bien recibidos por las comunidades y fueron un importante punto de partida para que en cada proyecto se buscara compartir los resultados de investigación con las comunidades a través de presentaciones verbales en reuniones y talleres, o a través de materiales escritos. Un reto aún vigente es investigar temas de interés para las comunidades locales, así como compartir la información científica generada. A las familias campesinas les 
Estación de Biología

Chamela UNAM
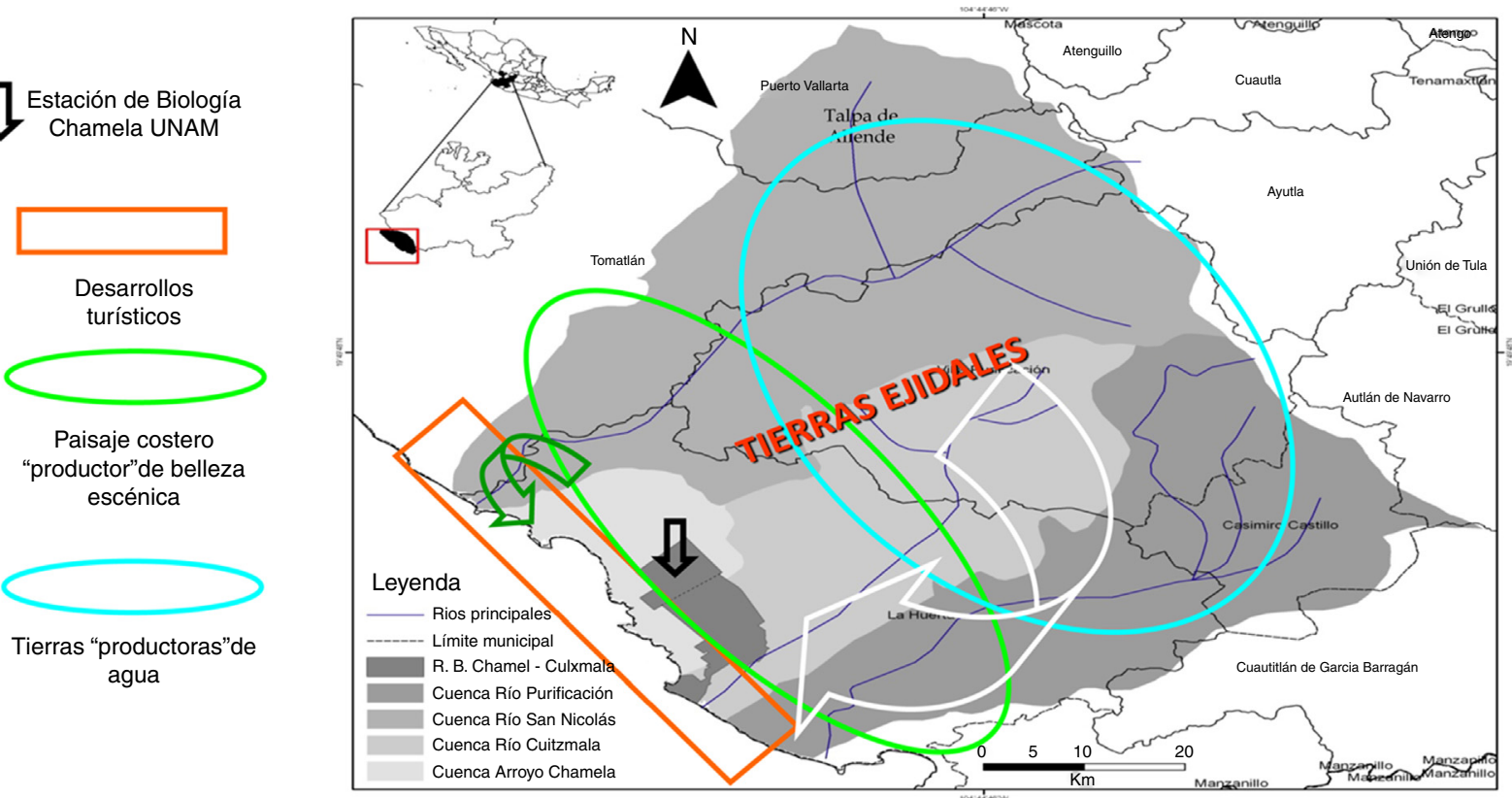

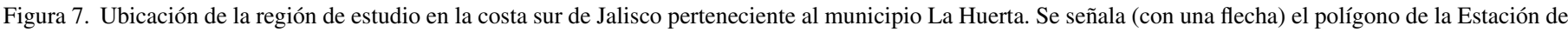

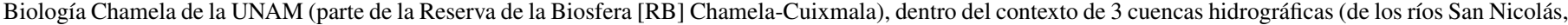

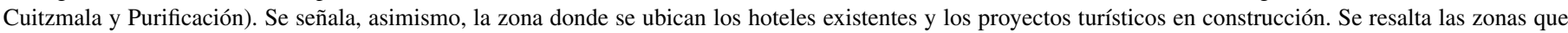

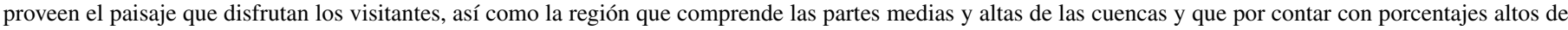

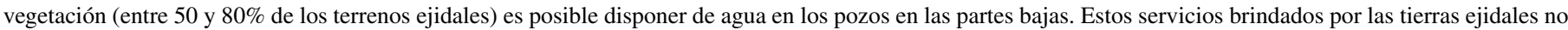
se reconocen y tampoco existen remuneración alguna. Figura realizada por Marcela Pérez.

interesa conocer y aprender sobre plantas y animales del bosque tropical seco y están ávidos de trabajar con la comunidad científica (Pérez-Escobedo, 2011). Este punto debe llevar a las instituciones académicas a reflexionar sobre qué y para qué investigar, así como construir estrategias de comunicación que apoyen las prácticas de aprovechamiento de recursos naturales, la conservación de ecosistemas y nutrir las políticas públicas. Nuestra aportación en este sentido ha sido por ahora publicar una serie de libros de divulgación «Las tierras y los montes de la costa de Jalisco» que consta de 5 volúmenes que se han trabajado en escuelas primarias, telesecundarias y con grupos de adultos (fig. 6). Tal proyecto aspira a dar pasos en esta dirección.

\section{Procesos educativos en el contexto de la ciencia para la sustentabilidad}

Los procesos educativos para la sustentabilidad deben considerar que el entendimiento de los sistemas complejos rebasa la visión de una disciplina y por ello los procesos multi-, intery transdisciplinarios son necesarios (García, 1994). También se requieren enfoques que flexibilicen los procesos de investigación. El entendimiento de un fenómeno es deseable para poder diseñar una acción transformadora, pero esta visión en fenómenos complejos resulta prácticamente imposible. La complejidad y la elevada incertidumbre de los sistemas socioecológicos requieren de quienes intentan entenderlos y manejarlos bajo formas de aproximación diferentes. El concepto de manejo adaptativo surge como una alternativa para entender un sistema, actuar sobre este, monitorear el efecto de las intervenciones y efectuar ajustes continuamente (Grumbine, 1994; Holling, 1978). El entendimiento y el diseño de acciones van a la par y el continuo monitoreo forma parte del proceso de investigación (Folke et al., 2004; Grumbine, 1994). Construir ciencia para la sustentabilidad requiere entonces formar profesionistas dispuestos a valorar distintas disciplinas y que promuevan su interacción (Clark et al., 2016; García, 1994), para hacer operativas las acciones de manejo adaptativo.

Un proyecto educativo al que haremos referencia tiene su origen en un curso de posgrado que se ha llevado a cabo por 11 años consecutivos en Warmiragra, en los Andes Centrales de Perú. El esquema de trabajo se basa en la colaboración con comunidades quechúas de la zona, organizaciones civiles (fig. 8) y las escuelas locales, en múltiples temas que incluyen organización territorial, sistemas agrícolas, pecuarios y forestales, recursos genéticos, flora y fauna. Este trabajo es parte del curso de campo «Domesticación y manejo in situ de recursos genéticos» realizado por el Posgrado en Ciencias Biológicas de la UNAM y la Escuela de Posgrado de la Universidad Nacional Agraria La Molina de Perú, en el que además participan continuamente miembros de organizaciones civiles como GEA y CADEP y organizaciones gubernamentales como la Conabio, México y el MINAM, Perú, entre otras (fig. 8).

El otro proyecto se lleva a cabo en la licenciatura en Ciencias Ambientales (LCA) de la UNAM, en la cual se ha estimulado la interdisciplina y la capacidad de valorar y aprender de otros sectores de la sociedad en la búsqueda de soluciones a problemas. Junto con GEA, la organización campesina Sansekan Tinemi y comunidades rurales nahuas y mestizas de Chilapa y Ahuacuotzingo, en la región centro montaña de Guerrero 


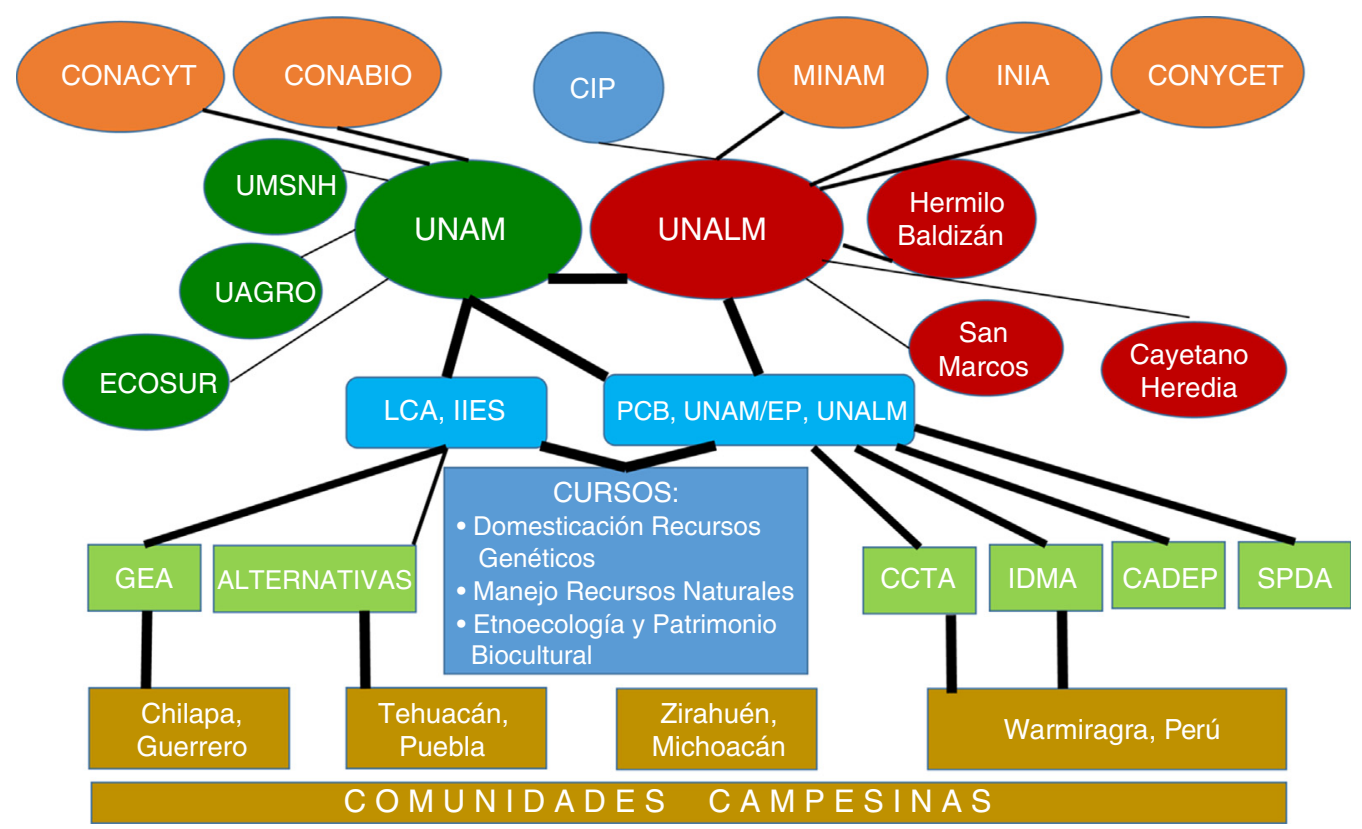

Figura 8. Esquema general de los procesos educativos para la participación que impulsa nuestro grupo de trabajo. En un plano general destaca la interacción entre la Universidad Nacional Autónoma de México (UNAM) y la Universidad Nacional Agraria la Molina (UNALM), de Perú, las cuales a través del Posgrado en Ciencias Biológicas y la Escuela de Posgrado de ambas instituciones, respectivamente, han impulsado por 11 años consecutivos el curso «Domesticación y manejo de recursos genéticos». Este curso cubre de manera intensiva 40 horas de teoría, así como 10 horas para el diseño de proyectos de investigación sobre diversos temas relacionados con el manejo de recursos naturales, sistemas de producción, uso del territorio, entre una amplia gama de problemas ambientales que se viven en comunidades de la región central de Los Andes peruanos, ubicadas en la caña de Warmiragra y que año tras año se van registrando con base en las necesidades locales. Los proyectos de investigación se comparten y realizan con la participación de los miembros de las comunidades. Los informes técnicos y materiales didácticos que resultan del trabajo se entregan al año siguiente a las escuelas de las localidades de Armatanga y Chinchubamba, así como a las ONG que participan localmente (Instituto de Desarrollo y Medio Ambiente (IDMA) con sede en Huánuco, Perú) y la Coordinadora de Ciencia y Tecnología Andina (CCTA), la cual colabora en la organización y realización de los cursos. Ambas ONG son puentes indispensables para la realización de las investigaciones e interrelaciones con las comunidades andinas. Colabora con el curso, continuamente, la Sociedad Peruana de Derecho Ambiental (SPDA), cuyos integrantes participan en el abordaje de temas sobre derechos de propiedad intelectual y diversos aspectos de legislación ambiental. Asimismo, los integrantes del Grupo de Estudios Ambientales de México y el Centro Andino de Capacitación y Promoción José María Arguedas (CADEP) envían anualmente miembros de las organizaciones al curso. CADEP, con sede en Cusco organiza frecuentemente nuestra participación en versiones cortas del curso para sus promotores. A este proyecto iniciado por la UNAM y la UNALM se han sumado paulatinamente la Universidad Nacional Hermilo Valdizán de Huánuco y la Universidad Nacional de San Marcos, y la Universidad Cayetano Heredia de Lima, Perú. El curso recibe apoyo financiero del Conycet de Perú y Conacyt, México, así como la colaboración de instituciones gubernamentales de Perú (Ministerio del Ambiente, MINAM e Instituto Nacional de Investigación Agraria, INIA) y de México (particularmente, la Comisión Nacional para el Conocimiento y Uso de la Biodiversidad, Conabio). Finalmente, desde sus inicios destaca la colaboración del Centro Internacional de la Papa (CIP), un organismo internacional que presenta en el curso un panorama del quehacer institucional. Con base en la experiencia internacional en Perú, se ha puesto en práctica por 5 generaciones consecutivas un esquema de trabajo similar, impulsado por el Instituto de Investigaciones en Ecosistemas y Sustentabilidad (IIES), en conjunto con alumnos de la licenciatura en Ciencias Ambientales (LCA) en Chilapa, Guerrero con la colaboración del Grupo de Estudios Ambientales, en el Valle de Tehuacán-Cuicatlán con el apoyo de Alternativas, A.C. y organizaciones comunitarias de Zapotitlán de las Salinas, San Juan Raya y Santiago Tilapa, Puebla, así como en Zirahuén, Michoacán. En estos casos las investigaciones van precedidas de asambleas comunitarias que discuten y proponen los temas de investigación, se involucran en el diseño y realización y reciben de la siguiente generación los informes técnicos y materiales de difusión elaborados por la generación previa.

desarrollamos por 6 años consecutivos una experiencia de investigación para desarrollar acciones ante problemas ambientales (Lindig y Casas, 2013).

El trabajo ha comprendido la integración de los temas del último semestre de la carrera: 1) aprovechamiento de recursos naturales y servicios ecosistémicos, 2) biología de la conservación, 3) restauración ecológica y 4) ordenamiento territorial. Una comisión de profesores y alumnos ha acudido sistemáticamente a reuniones con GEA y con representantes de las comunidades incluidas en la organización (32 comunidades). En esas reuniones se expone información sobre el programa educativo de la UNAM, los resultados de estudios del año anterior, la intención de contribuir con investigaciones útiles a las comunidades y a GEA durante el año en turno, y se solicita permiso para trabajar en sus comunidades. Por su parte, GEA y las comunidades exponen sus propias agendas de necesidades de información y se deciden los proyectos a realizar y las comunidades en las que se trabajará cada uno de ellos. Durante el periodo de trabajo se han llevado a cabo más de 20 proyectos de investigación (tabla 1). Algunos de estos han sido específicos para un año en particular, otros como los inventarios de recursos forestales, el estudio de sistemas agroforestales, consumo de leña y diagnóstico de cantidad y calidad de agua y riesgos asociados a deslizamientos, han tenido continuidad por varios años. Los objetivos del trabajo son: 1) realizar investigaciones cortas que permitan a los alumnos visualizar el valor de la investigación en procesos para la atención de problemas, 2) vincularse con diferentes sectores de la comunidad, con GEA como organización civil y eventualmente con autoridades regionales, comerciantes, entre otros sectores y 3 ) adquirir una identidad y compromiso insti- 
Tabla 1

Principales proyectos de investigación llevados a cabo conjuntamente entre alumnos de la licenciatura en Biología y maestría en Ecología Aplicada de la Escuela de Posgrado y del Posgrado en Ciencias Biológicas de la UNAM, con campesinos y organizaciones civiles en Warmiragra, Perú. Proyectos de los alumnos de la licenciatura en Ciencias Ambientales, UNAM, en Chilapa, Gro., Tehuacán, Pue., y Zirahuén, Mich, en conjunto con organizaciones sociales, comunidades rurales y organizaciones civiles trabajando en las zonas.

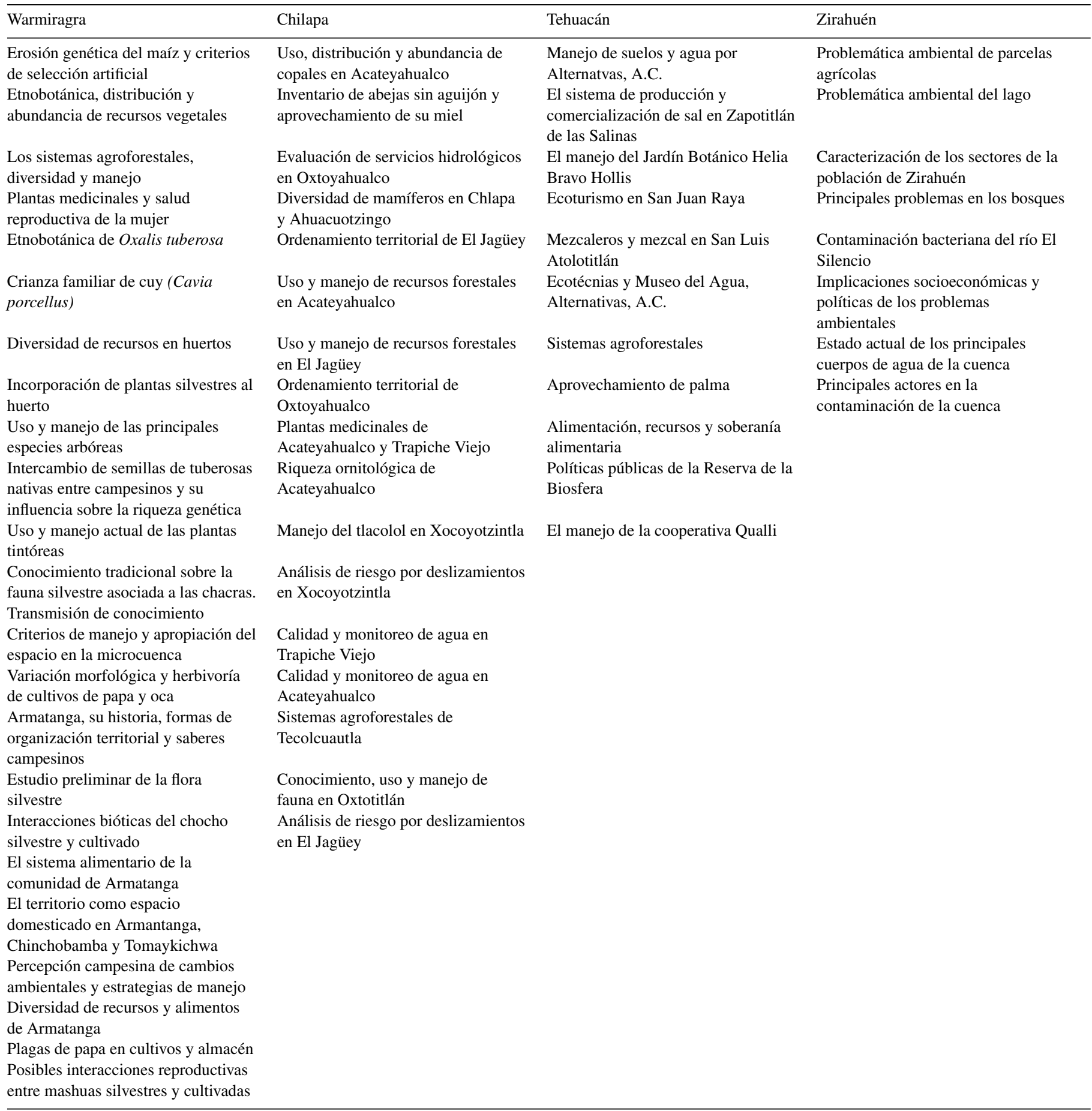

tucional como académicos en la colaboración que se desarrolla (fig. 8). El compromiso de los universitarios es entregar informes técnicos, materiales de difusión, colecciones, materiales educativos, manuales, mapas, y otros productos que son de utilidad a las comunidades en sus decisiones y construcción de acuerdos. También son útiles a GEA en el diseño de su agenda de trabajo.
Se designa a un equipo de alumnos y asesores (profesores y miembros de GEA) la responsabilidad de diseñar los protocolos de investigación solicitados, efectuarlos en campo, e integrar un primer borrador del informe técnico. La siguiente generación de alumnos comienza su ejercicio revisando el informe técnico de la generación previa, elaborando los materiales de difusión y 
atendiendo el diseño e investigación del nuevo ciclo, así como el nuevo informe técnico. De esta manera, cada generación retoma sucesivamente el trabajo inconcluso de la generación previa, lo termina y deja el nuevo trabajo a la próxima generación. Ello genera un sentimiento de identidad sectorial y compromiso con los otros sectores. El proyecto se convierte así, más que en una práctica escolar en un proceso de compromiso institucional en la atención de problemas reales.

La LCA ha llevado a cabo múltiples prácticas de integración como ejercicios que pretenden estimular en los estudiantes la incorporación de referentes conceptuales y metodológicos de distintas disciplinas ante problemas comunes. Adicionalmente, generan información científica que puede proveer a los actores relacionados con un problema ambiental elementos de juicio para tomar decisiones. Con el tiempo, estos ejercicios se han transformando en un espacio para la construcción de marcos conceptuales de investigación en ciencias ambientales, que permiten la formación de los futuros científicos y que aportan información para el entendimiento y la posible solución de problemas ambientales concretos. Un espacio de trabajo recurrente de la LCA es el lago y el poblado de Zirahuén. La confluencia de actores sociales relacionados de manera diferencial con múltiples problemas ambientales hace de la región un espacio de trabajo de gran interés. Los resultados de las primeras prácticas de la LCA han permitido caracterizar los problemas e indican un acelerado deterioro ambiental en los últimos años. Entre los problemas reportados se encuentran la deforestación, el cambio de uso de suelo, la contaminación de cuerpos de agua, y disputas por el territorio y sus recursos. La naturaleza compleja de estos problemas involucra la interacción de componentes biofísicos, económicos, sociales, políticos y culturales y la mutua dependencia entre estos. Como un primer acercamiento al estudio la problemática ambiental en Zirahuén, desde una perspectiva integral e interdisciplinaria, profesores y estudiantes de la LCA y los pobladores, propusimos el desarrollo de un diagnóstico ambiental. Este trabajo partió de la solicitud de las comunidades indígenas de la región, como base para encontrar soluciones a problemas ambientales. Derivado del primer ejercicio, se desarrolló una segunda etapa de trabajo en la que se profundizó en la problemática del agua y en la ampliación de la escala de estudio a nivel de cuenca hidrológica. Se ha trabajado en conjunto con las autoridades locales y se pretende llevar un proceso institucional que logre una relación a largo plazo para continuar la formación académica de los alumnos y de seguimiento a los problemas y posibles soluciones. En la educación para la ciencia de la sustentabilidad y en la actualización y formación de los profesores es fundamental el desarrollo de ejercicios en los lugares donde se desarrollan los problemas ambientales. En el caso de la enseñanza de la complejidad es indispensable realizar estos ejercicios como lo es el escenario de Zirahuén.

En el caso de Warmiragra Perú, el proceso comunicativo ha involucrado la escala de cuenca. Ahí la plataforma que ha detonado comunicación entre comunidades campesinas ONG y organizaciones académicas han sido las escuelas locales. Se inició la realización de la Feria Ambiental de Warmiragra que ha impulsado exitosamente la convocatoria a niños, jóvenes y adultos de las comunidades, las ONG regionales y las universidades participantes un proceso de intercambio de saberes a través de talleres, actividades lúdicas e intercambios de experiencias que buscan proyectar su impacto a mayor escala.

\section{Procesos institucionales de comunicación y participación}

Poco después de su creación en 2003, el IIES, de la UNAM decidió de manera colegiada la creación de una Unidad de Vinculación que facilitara los procesos de comunicación entre los grupos de investigación de esta entidad académica y los distintos sectores con los que colaboran tales grupos. Pero también se concibió que el proceso de vinculación debería adoptarse como un proyecto institucional, más allá de la iniciativa personal de algunos académicos. El planteamiento general es que la vinculación es un mecanismo fundamental para articular la investigación en temas ambientales con aquellos sectores que viven y afrontan los problemas. De tal manera, la agenda de investigación puede y debe construirse a partir de la problemática concreta del entorno, de las regiones y de los sectores con los cuales trabajan los grupos de investigación. Esto implica asumir un compromiso social de la investigación científica. Además, contempla la idea de que la investigación que involucra los sectores sociales con los cuales se interactúa permite una construcción social del conocimiento consecuente con las premisas de la transdisciplina para la ciencia para la sustentabilidad. La magnitud de la demanda de apoyo en comunicación e investigación ambiental rápidamente se elevó desde sectores sociales, empresariales organizaciones sociales, organizaciones civiles e instancias gubernamentales desde la escala comunitaria, municipal, estatal y nacional (fig. 9). De tal forma, la Unidad de Vinculación enfrentó problemas organizativos y las actividades de vinculación tuvieron que replantearse para incluir no solo a esta unidad, son también a la Unidad de Ecotecnologías que tiene interacciones con diversos sectores de la sociedad, y la Unidad del Ecojardín del IIES que anualmente atiende a miles de visitantes al campus Morelia de la UNAM. Adicionalmente, los grupos de investigación progresivamente han venido fortaleciendo sus propias capacidades y medios para atender aspectos de difusión y vinculación en la práctica de investigación con los sectores con los que interactúan. La idea de la Unidad de Vinculación ha venido desarrollándose con los años. Actualmente, el IIES considera la vinculación con la sociedad como una actividad sustantiva, pues el tipo de problemas de investigación que aborda así lo amerita. Para ello ha concebido a la Unidad de Vinculación y a las otras unidades mencionadas como facilitadoras de procesos de interacción entre los propios grupos de trabajo del IIES, la institución en general así como entre éstos y otros sectores de la sociedad. El IIES ha incluido en sus programas de estímulos académicos criterios que valoran las labores de vinculación a los integrantes de los grupos de trabajo. Se trata entonces de articular distintas plataformas de trabajo que están abordando en sus agendas de investigación problemas ambientales. Permiten involucrar de manera creciente la investigación de problemas particulares para apoyar el desarrollo de regulaciones (como en el caso ilustrado con A. potatorum), así como la atención de programas municipales y estatales que solicitan asesorías ante problemas como ordenamiento territo- 


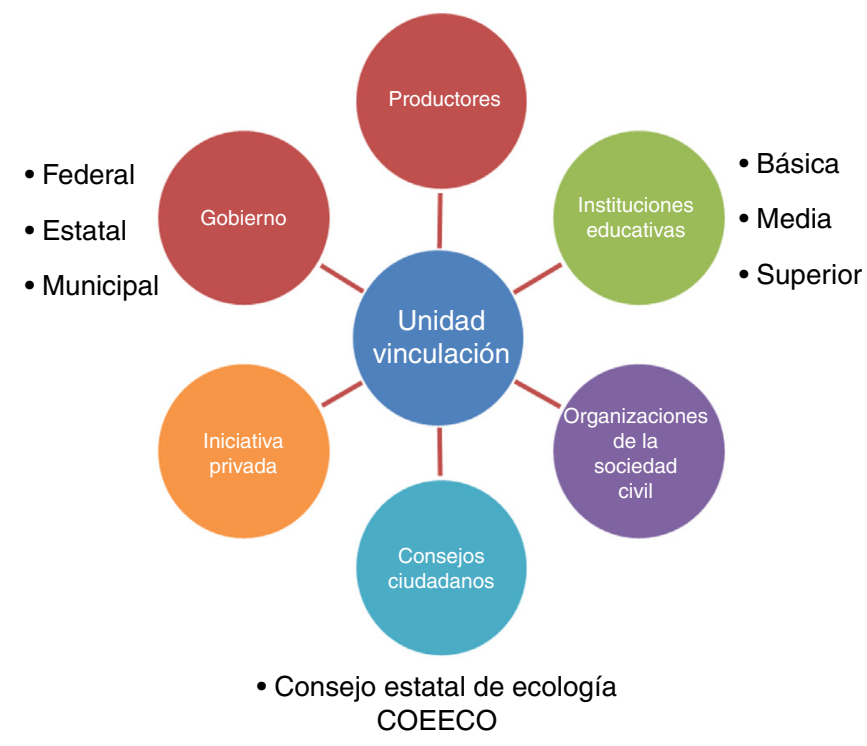

Figura 9. Las interrelaciones de la Unidad de Vinculación del Instituto de Investigaciones en Ecosistemas (IIES), UNAM con otros sectores de la sociedad regional y nacional. A pesar de las dificultades organizacionales, las Unidades de Apoyo Académico como la Unidad de Ecotecnologías, Ecojardín y la Unidad de Vinculación, han logrado desarrollar importantes proyectos colaborativos interinstitucionales. Por ejemplo, existe una importante relación de colaboración entre el sector educativo y la Unidad de Vinculación, así como con dependencias del sector ambiental como Conanp, Conabio y Conafor. A nivel municipal, el IIES fue impulsor del programa «Agenda desde lo local» y a nivel estatal el IIES es parte del Consejo Estatal de Ecología y participa activamente en los espacios de consulta para el diseño de política ambiental. También es importante reconocer que los investigadores realizan actividades de vinculación particularmente con productores, comunidades y empresas, fortaleciendo de esta forma la atención de problemas relacionados con la producción agrícola, pecuaria y forestal.

rial, manejo de agua, manejo forestal, entre otros. Y, finalmente, está la escala de problemas nacionales de los cuales instancias gubernamentales y organizaciones civiles también solicitan el apoyo del IIES o de grupos de investigación del IIES. Son ejemplos de estas demandas, la evaluación de proyectos nacionales como el programa PROÁRBOL, el Programa de Pago por Servicios Ambientales, el proyecto Capital Natural de México desde la Conabio, el diagnóstico de plantas malezas e invasoras, entre otros programas que requieren evaluaciones técnicas y propuestas.

Las actividades de vinculación de los grupos y unidades del IIES comprenden una amplia gama de actividades. Incluye la divulgación de la ciencia a través de medios masivos de comunicación y la atención directa al público a través de conferencias y ferias de ciencia, puertas abiertas y otras iniciativas en plazas y calles. También incluye la atención de problemas ciudadanos en su agenda de investigación e innovación tecnológica. Y finalmente, el vínculo con los tomadores de decisiones a distintos niveles y la procuración por incidir en políticas públicas, también a distintas escalas. En todas estas acciones se parte de considerar que los temas ambientales no pueden soslayar los diferentes sectores que enfrentan la problemática ambiental ni la toma de decisiones al respecto; por lo tanto, la vinculación más allá de las iniciativas de los individuos debe incluir a las instituciones.

\section{Discusión y perspectivas}

La ciencia para la sustentabilidad es un paradigma de extraordinaria importancia y su construcción debe ser una prioridad para los grupos de investigación y las instituciones que abordan la comprensión y atención de problemas ambientales (Kates, 2011; Kates et al., 2001). Requiere impulsar en la práctica de investigación una renovada disposición a considerar de manera horizontal los conocimientos y experiencias técnicas que los diferentes grupos humanos han construido a lo largo de siglos o milenios. Requiere una apertura a nuevos esquemas de innovación tecnológica basada en la consideración de la fuente múltiple de conocimientos y técnicas, y requiere la apertura al ensayo y error que involucra el concepto de manejo adaptativo. Este concepto ha sido considerado por las visiones tradicionales de la ciencia como una carga de irresponsabilidad. Construir manejo adaptativo significa asimilar los conocimientos disponibles para la atención de un problema con el mismo rigor o aún mayor que las investigaciones en campos específicos. El rigor debe ser mayor pues conlleva tras de sí la conciencia de la consecuencia de que la acción tendrá una repercusión sobre la sociedad y/o los ecosistemas involucrados. Pero se trata también de incorporar el monitoreo de las acciones como parte del proceso de investigación. Este proceso no excluye el rigor científico, por el contrario, lo demanda con mayor responsabilidad y principios éticos.

Construir ciencia para la sustentabilidad implica formar científicos con nuevas visiones. La complejidad de los sistemas que deben abordarse requiere una amplia visión y buena disposición a la interacción disciplinaria. Las visiones simplistas sobre ciencias «duras» $\mathrm{y}$ «blandas» carecen de valor en esta nueva búsqueda de aproximaciones de mayor apertura. Los científicos naturales deben ser capaces de valorar el aporte de las ciencias sociales y viceversa. No hay ciencia de primera o de segunda categoría, cada campo tiene sus aportes y todos ellos son de alto valor para la solución de problemas. De manera similar, los científicos básicos deben tener la apertura necesaria para valorar la ciencia aplicada y los procesos de innovación tecnológica en una dimensión similar. No hay ciencias mejores que otras, son campos científicos distintos y la pluralidad de enfoques es más que nunca necesaria. La formación de profesionistas preparados para las interacciones disciplinarias y capaces de valorar el conocimiento de otros sectores de la sociedad (investigación transdisciplinaria) debe consolidarse. No se trata de contraponer el valor de las disciplinas que se han desarrollado a lo largo de la historia de la ciencia, sino de articularlas, de orquestarlas para atender problemas a los que por sí mismas solo pueden dar respuestas parciales. Ello implica hacer ajustes curriculares en diversos programas académicos, pero también en las instituciones que los pondrán en práctica. Existen ya experiencias en desarrollo que permiten analizar las lecciones que brindan los esfuerzos educativos multi- e interdisciplinarios. Lo hemos analizado ahora desde la perspectiva de las ciencias ambientales, pero en realidad son múltiples los campos que requieren cada 
vez más la visión de la complejidad y la necesidad de trabajo de investigación interdisciplinaria.

Finalmente, es preciso señalar que las iniciativas individuales de investigadores y ciudadanos preocupados por los problemas ambientales que imperan en esta gran crisis socioecológica son todas de gran valor. Sin embargo, es tiempo de que las instituciones construyan sus agendas para dar atención colectiva a problemas que rebasan las posibilidades de los individuos. Es este quizás el mayor reto, pues implica poner en sintonía instituciones gubernamentales, no gubernamentales, organizaciones sociales, e instituciones académicas a distintas escalas para facilitar su interacción. Se trata de grandes retos organizacionales, operativos que involucran la valoración de la participación para la construcción de una vigorosa ciencia para la sustentabilidad.

\section{Agradecimientos}

Los autores agradecen el apoyo de diversos proyectos de investigación DGAPA, PAPIIT UNAM proyecto IN209214, PAPIME PE304315 y PE207813, Conacyt CB-2013-01221800, así como la colaboración de organizaciones civiles (en especial GEA, Contec, México y CCTA, IDMA, CADEP, Perú), organizaciones sociales (Sansekan Tinemi) y numerosas comunidades campesinas del valle de Tehuacán-Cuicatlán, México, Zirahuén, Cuiteco, la montaña de Guerrero, México y la región de la cuenca de Warmiragra en los Andes Centrales de Perú, particularmente a la familia Ticlavilca. Asimismo, el apoyo académico de grupos de investigación del IIES, UNAM, el CIZA de la Universidad Nacional Agraria La Molina, Perú.

\section{Referencias}

Adames-Mayorga, E. (2007). Hegemonía y cultura científica. Base para un debate entre ciencias. Tareas, 125, 5-28.

Alcorn, J. B. (1993). Indigenous people and conservation. Conservation Biology, 7, 424-426.

Altieri, M. y Toledo, V. M. (2011). The agroecological revolution in Latin America: rescuing nature, ensuring food sovereignty and empowering peasants. Journal of Peasant Studies, 38, 587-612.

Barnosky, A. D., Hadly, E. A., Bascompte, J., Berlow, E. L., Brown, J. H., Fortelius, M., et al. (2012). Approaching a state shift in Earth's biosphere. Nature, 486, 52-58.

Berkes, F. (1999). Sacred ecology: traditional ecological knowledge and resource management. Philadelphia: Taylor \& Francis.

Berkes, F. y Berkes, M. K. (2009). Ecological complexity, fuzzy logic and holism in indigenous knowledge. Futures, 41, 6-12.

Berkes, F., Colding, J. y Folke, C. (2000). Rediscovery of traditional ecological knowledge as adaptive management. Ecological Applications, 10, $1251-1262$.

Boege, E. (2008). El patrimonio biocultural de los pueblos indígenas de México. México D.F: Instituto Nacional de Antropología e Historia: Comisión Nacional para el Desarrollo de los Pueblos Indígenas.

Boege, K., Castillo, A., García, A., Vega, J. H., Miranda, A., Ruiz, A., et al. (2010). Dictamen técnico de la Manifestación de Impacto Ambiental del proyecto de desarrollo turístico Zafiro (clave 14JA2009T0017): identificación de posibles impactos a las áreas naturales protegidas de la región. Ciudad de México: Comité Técnico Asesor de la Reserva de la Biosfera Chamela Cuixmala, UNAM /Fundación Ecológica de Cuixmala, A.C.

Buscaglia, E. (2015). Lavado de dinero y corrupción política. El arte de la delincuencia organizada internacional. México D.F: Penguin Random House Grupo Editorial, S.A. de C.V.
Camou-Guerrero, A. (2008). Los recursos vegetales en una comunidad rarámuri: aspectos culturales económicos y ecológicos (Tesis doctoral). Morelia, Michoacán: Posgrado en Ciencias Biológicas, Universidad Nacional Autónoma de México.

Camou-Guerrero, A., Reyes-García, V., Martínez-Ramos, M. y Casas, A. (2008). Knowledge and use value of plant species in a Rarámuri community: a gender perspective for conservation. Human Ecolology, 36, 259-272.

Castillo, A. (1999). La educación ambiental y las instituciones de investigación ecológica: hacia una ciencia con responsabilidad social. Tópicos en Educación Ambiental, 1, 35-46.

Castillo, A., Domínguez, C., García, A., Quesada, M. A. y Vega, J. H. (2007). Proyectos de desarrollo turísticos La Huerta (Clave: 14JA2006T0018 (Marina Careyes) y La Tambora (Clave: 14JA20-06T0011) en las áreas vecinas de la Reserva de la Biosfera Chamela-Cuixmala. Ciudad de México: UNAM

Castillo, A., Godínez, C., Schroeder, N., Galicia, C., Pujadas-Botey, A. y Martínez, L. (2009). . pp. 844-850. Los bosques tropicales secos en riesgo: conflictos entre uso agropecuario desarrollo turístico y provisión de servicios ecosistémicos en la costa de Jalisco (34) México: Interciencia.

Castillo, A., Pujadas, A., Magaña, M. A., Martínez, L. y Godínez, C. (2006). Comunicación para la conservación: análisis y propuestas para la Reserva de la Biosfera Chamela-Cuixmala Jalisco. En A. Barahona y L. Almeida (Eds.), Educación para la conservación (pp. 93-109). México D.F: Facultad de Ciencias y Programa Universitario de Medio Ambiente, UNAM.

CCA (Comisión para la Cooperación Ambiental de América del Norte). (2005). Expediente de hechos final. Petición ciudadana Tarahumara. SEM-00-006. Derecho y políticas ambientales en América del Norte (DPAAN). Montreal: CCA.

Chambers, R. (1983). Rural development. Putting the last first. Essex, Inglaterra: Longmans Scientific and Technical Publishers.

Clark, W. C. (2007). Sustainability science: a room of its own. Proceedings of the National Academy of Science, 104, 1737-1738.

Clark, W. C. y Dickson, N. M. (2003). Sustainability science: the emerging research program. Proceedings of the National Academy of Science USA, 100, 8059-8061.

Clark, W. C., Lorrae van Kerkhoff, L. L. y Gallopin, G. (2016). Crafting usable knowledge for sustainable development. HKS Faculty Research Working Paper Series RWP16-005. Cambridge, Massachusetts: Harvard Kennedy School.

Cohen, D. (2014). Estrategias de manejo del bosque tropical seco: un estudio de caso en Jalisco (Tesis). Morelia, Michoacán: Licenciatura en Ciencias Ambientales, Escuela Nacional de Estudios Superiores Universidad Nacional Autónoma de México.

Comisariado Ejidal de Cuiteco. (2007). Acta de asamblea del ejido Cuiteco. Cuiteco, Chihuahua, México.

Cornell, S., Berkhout, F., Tuinstra, W., Tàbara, J. D., Jäger, J., Chabay, I., et al. (2013). Opening up knowledge systems for better responses to global environmental change. Environmental Science \& Policy, 28, 60-70.

Delgado-Lemus, A., Casas, A. y Téllez, O. (2014). Distribution, abundance and traditional management of Agave potatorum in the Tehuacán Valley Mexico: bases for sustainable use of non-timber forest products. Journal of Ethnobiology and Ethnomedicine, 10, 63.

Delgado-Lemus, A., Torres, I., Blancas, J. y Casas, A. (2014). Vulnerability and risk management of Agave species in the Tehuacán Valley, Mexico. Journal of Ethnobiology and Ethnomedicine, 10, 53.

Estrella-Ruiz, P. (2008). Efecto de la explotación humana en la biología de la polinización de Agave salmiana y Agave potatorum en el Valle de Tehuacán-Cuicatlán (Tesis de maestría). México, D.F.: Posgrado en Ciencias Biológicas, Universidad Nacional Autónoma de México.

Félix-Valdez, L., Vargas-Ponce, O., Cabrera-Toledo, D., Casas, A., CibriánJaramillo, A. y Cruz-Larios, L. (2015). Effects of management for mescal production on the diversity and genetic structure of Agave potatorum Zucc., in Central Mexico. Genetic Resources and Crop Evolution, 63, 1255-1271.

Folke, C., Carpenter, S., Walker, B., Scheffer, M., Elmqvist, T., Gunderson, L., et al. (2004). Regime shifts, resilience, and biodiversity in ecosystem management. Annual Review of Ecology, Evolution, and Systematics, 35, $557-581$.

Freire, P. (1973). ¿Extensión o comunicación? México D.F: Siglo XXI Editores. 
Funtowicz, S. O. y Ravetz, J. R. (1993). Science for the post-normal age. Futures, 25, 739-755.

García, R. (1994). Interdisciplinariedad y sistemas complejos. En E. Leff (Ed.), Ciencias sociales y formación ambiental (pp. 85-124). Barcelona: Gedisa.

Gibbons, M. (2000). Mode 2 society and the emergence of context-sensitive science. Science and Public Policy, 27, 159-163.

Gibbons, M., Limoges, C., Nowotny, H., Schwartzman, S., Scott, P. y Trow, M. (1994). The new production of knowledge: the dynamics of science and research in contemporary societies. Londres: Sage.

Grumbine, R. E. (1994). What is ecosystem management? Conservation Bio$\log y, 8,27-38$.

Holling, C. S. (1978). Adaptive environmental assessment and management. Chichester, UK: John Wiley and Sons.

Kates, R. W. (2011). What kind of science is sustainability science? Proceedings of the National Academy of Science, 108, 19449-19450.

Kates, R. W., Clark, W. C., Corell, R., Hall, J. M., Jaeger, C. C., Lowe, I., et al. (2001). Sustainability science. Science, 292, 641-642.

Klein, J., Grossenbacher-Mansuy, W., Häberli, R., Bill, A., Scholz, R. W. y Welti, M. (2001). Transdisciplinarity: joint problem solving among science. Technology, and society. An effective way for managing complexity. Basel: Birkhauser Verlag [consultado 8 Jul 2016]. Disponible en: http://tocs.ulb.tudarmstadt.de/95828303.pdf.

Lang, D. J., Wiek, A., Bergmann, M., Stauffacher, M., Martens, P., Moll, P., et al. (2012). Transdisciplinary research in sustainability science: practice, principles, and challenges. Sustainability Science, 7, 25-43.

Lewis, M., Gary, P., Simons, F. y Fennig, C. D. (2015). Ethnologue: languages of the World, Eighteenth edition. Dallas: SIL International [consultado 8 Jul 2016]. Versión digital disponible: http://www.ethnologue.com.

Lindig, R. y Casas, A. (2013). Experiencias docentes en integración de conocimientos ambientales en comunidades rurales. En A. Camou-Guerrero, A. Castillo, y E. García-Frapolli (Eds.), Procesos de formación educativa interdisciplinaria: miradas desde las Ciencias Ambientales (pp. 125-144). Morelia, Michoacán: CIEco, UNAM.

Maffi, L. (2007). Biocultural diversity and sustainability. En J. Pretty, A. Ball, T. Benton, J. Guivant, D. R. Lee, y D. Orr, et al. (Eds.), Ward H. handbook of environment and society (pp. 267-277). Londres: Sage.

Maffi, L. y Woodley, E. (2010). Biocultural diversity conservation: a global sourcebook. New York: Earthscan/Routledge.

Martínez-Alier, J. (2015). Macroeconomía ecológica, metabolism social y justicia ambiental. En M. Imaz (Ed.), El pensamiento ecológico frente a los retos del siglo XXI (pp. 47-87). México D.F: Universidad Nacional Autónoma de México.

MEA (Millennium Ecosystem Assessment). (2005). Ecosystems and human well-being. Biodiversity synthesis. Washington D.C.: World Resources Institute.

Noguera, F., Vega, J. H. y Aldrete, A. N. (2002). Introducción. En F. A. Noguera, J. H. Vega, A. N. García, y M. Quesada-Avendaño (Eds.), Historia natural de Chamela (pp. $x v$-xxi). México D.F: Instituto de Biología, Universidad Nacional Autónoma de México.

OXFAM. (2012). Crisis, inequality and poverty. Intermón OXFAM briefing papers 32 [consultado 8 Jul 2016]. Disponible en: http://www. oxfamintermon.org/sites/default/files/documentos/files/Oxfam\% 20Intermon\%20report\%2032\%20EN.pdf.

Pérez-Escobedo, H. M. (2011). Necesidades de información para el manejo de los socio-ecosistemas de la región Chamela-Cuixmala, Jalisco. México D.F: Posgrado en Ciencias Biológicas, Universidad Nacional Autónoma de México.
Pujadas, A. (2003). Comunicación y participación social en el programa de Ordenamiento Ecológico Territorial de la Costa de Jalisco y la Reserva de la Biosfera Chamela-Cuixmala (Tesis de maestría). Morelia, Michoacán: Posgrado en Ciencias Biológicas Universidad Nacional Autónoma de México.

Rangel-Landa, S., Casas, A. y Dávila, P. (2015). Facilitation of Agave potatorum: an ecological approach for assisted population recovery. Forest Ecology and Management, 347, 57-74.

Riensche, M., Castillo, A., Flores-Díaz, A. y Maass, M. (2015). Tourism at Costalegre, Mexico: an ecosystem services-based exploration of current challenges and alternative futures. Futures, 66, 70-84.

Röling, N. (1990). Extension science: information systems in agricultural development. Cambridge: Cambridge University Press.

Scholz, R. W. y Steiner, G. (2015). The real type and ideal type of transdisciplinary processes: part I-theoretical foundations. Sustainability Science, 10, 527-544.

Scott, A. J. (1996). Regional motors of global economy. Futures, 28 , 391-411.

Steffen, W., Broadgate, W., Deutsch, L., Gaffney, O. y Ludwig, C. (2015). The trajectory of the Anthropocene: the great acceleration. The Anthropocene Review, 2, 81-98.

Steffen, W., Crutzen, P. J. y McNeill, J. R. (2007). The Anthropocene: are humans now overwhelming the great forces of nature AMBIO. A Journal of the Human Environment, 36, 614-621.

Tarrés, M. L. (Ed.). (2004). Observar, escuchar y comprender. Sobre la tradición cualitativa en la investigación social. México D.F.: FLACSO, El Colegio de México, Miguel Ángel Porrúa.

Tengö, M., Brondizio, E. S., Elmqvist, T., Malmer, P. y Spierenburg, M. (2014). Connecting diverse knowledge systems for enhanced ecosystem governance: the multiple evidence base approach. Ambio, 43, 579-591.

Toledo, V. M. (2002). Ethnoecology: a conceptual framework for the study of indigenous knowledge of nature. En J. R. Stepp, F. S. Wybdgan, y R. K. Zager (Eds.), Ethnobiology and biocultural diversity (pp. 511-552). Georgia: International Society of Ethnobiology.

Toledo, V. M. y Barrera-Bassols, N. (2008). La memoria biocultural. La importancia ecológica de las sabidurías tradicionales. Barcelona: Ipn.

Torres, I., Casas, A., Delgado-Lemus, A. y Rangel-Landa, S. (2013). Aprovechamiento, demografía y establecimiento de Agave potatorum en el valle de Tehuacán, México: aportes etnobiológicos y ecológicos para su manejo sustentable. Zonas Áridas, 15, 1-16.

Torres, I., Casas, A., Vega, E., Martínez-Ramos, M. y Delgado-Lemus, A. (2015). Population dynamics and sustainable management of mescal Agaves in Central Mexico: Agave potatorum in the Tehuacán-Cuicatlán Valley. Economic Botany, 69, 26-41.

Valiente-Banuet, A., Solís, L., Dávila, P., Arizmendi, M. C., Silva, C., OrtegaRamírez, J., et al. (2009). Guía de la vegetación del valle de TehuacánCuicatlán. México D.F: UNAM/Conabio.

Van Kerkhoff, L. y Lebel, L. (2006). Linking knowledge and action for sustainable development. Annual Review of Environmental Resources, 3 , 445-477.

Vitousek, P. M. (1994). Beyond global warming: ecology and global change. Ecology, 75, 1861-1876.

Vitousek, P. M., D’Antonio, C. M., Loope, L. L. y Westbrooks, R. (1996). Biological invasions as global environmental change. American Scientist, 84, 468-478.

Vitousek, P. M., Mooney, H. A., Lubchenco, J. y Melillo, J. M. (1997). Human domination of Earth's ecosystems. Science, 277, 494-499. 